\title{
Effect of cellular rearrangement time delays on the rheology of vertex models for confluent tissues
}

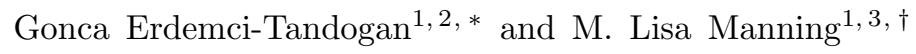 \\ ${ }^{1}$ Department of Physics, Syracuse University, Syracuse, NY 13244, USA \\ ${ }^{2}$ Institute of Biomedical Engineering, University of Toronto, Toronto, ON M5S 3G9, Canada \\ ${ }^{3}$ BioInspired Institute, Syracuse University, Syracuse, NY 13244, USA
}

\begin{abstract}
Large-scale tissue deformation during biological processes such as morphogenesis requires cellular rearrangements. The simplest rearrangement in confluent cellular monolayers involves neighbor exchanges among four cells, called a T1 transition, in analogy to foams. But unlike foams, cells must execute a sequence of molecular processes, such as endocytosis of adhesion molecules, to complete a T1 transition. Such processes could take a long time compared to other timescales in the tissue. In this work, we incorporate this idea by augmenting vertex models to require a fixed, finite time for $\mathrm{T} 1$ transitions, which we call the "T1 delay time". We study how variations in $\mathrm{T} 1$ delay time affect tissue mechanics, by quantifying the relaxation time of tissues in the presence of T1 delays and comparing that to the cell-shape based timescale that characterizes fluidity in the absence of any $\mathrm{T} 1$ delays. We show that the molecular-scale $\mathrm{T} 1$ delay timescale dominates over the cell shape-scale collective response timescale when the T1 delay time is the larger of the two. We extend this analysis to tissues that become anisotropic under convergent extension, finding similar results. Moreover, we find that increasing the T1 delay time increases the percentage of higher-fold coordinated vertices and rosettes, and decreases the overall number of successful T1s, contributing to a more elastic-like - and less fluid-like - tissue response. Our work suggests that molecular mechanisms that act as a brake on $\mathrm{T} 1$ transitions could stiffen global tissue mechanics and enhance rosette formation during morphogenesis.
\end{abstract}

\section{INTRODUCTION}

In processes such as development and wound healing, biological tissues must generate large-scale changes to the global shape of the tissue [1. In confluent tissues, where there are no gaps or overlaps between cells, such global changes necessarily correspond to either changes in individual cell shape or cell rearrangements [2, and largescale deformation almost always requires a large number of rearrangements.

For epithelial monolayers, the geometry of most such rearrangements is quite simple: viewing the apical side of the layer, four cells come together at a single four-fold vertex, which subsequently resolves into two three-fold vertices where cells have exchanged neighbors. This process is called a T1 transition, adopted from the literature on foams [3]. In some tissues, it is also common to observe higher-fold vertices called rosettes [4, 5].

Historically, there have been different perspectives on how to understand and quantify such rearrangements. At the molecular scale, a concerted sequence of processes must occur to allow such a change, including localization of non-muscle myosin and actin to shorten interfaces [6[10], unbinding of adhesion molecules and trafficking away from the membrane via endocytosis [11, exocytosis of adhesion molecules to newly formed interfaces and new homotypic binding, and reorganization of the cytoskeleton to stabilize the new edges. Moreover, molecules such as tricellulin [12, 13, are known to localize at tricellular junctions and must be reorganized [14, 15]. In addition to all this, there is recent evidence that some cell types possess mechanosensitive machinery that will only trigger this molecular rearrangement cascade if tension on the interface is sufficiently large [16].

A complementary perspective has focused on the collective behavior of cells in a tissue. Specifically, a combination of theoretical modeling [17, 18] and experimental data 19 21] has suggested that the collective mechanics of a tissue has a huge impact on the rate of cell rearrangements, and that the collective mechanics are dominated by a simple observable, the cell shape.

Shapes of cells in a confluent tissue are generated by a balance between contractility generated by the cytoskeleton and adhesion generated by molecules such as cadherins, as well as active force generation by cells [21, 22. This suggests that cell rearrangement rates are governed by cell-scale features, such as overall expression levels of adhesion and cytoskeletal machinery. Moreover, it is possible to identify a "collective response" timescale $\tau_{\alpha 0}[23$ that describes the typical timescale over which cells change neighbors. In both isotropic and anisotropic tissues, this timescale depends on cell shape and alignment [19, 21, 22.

Given the strong experimental support for cell-scale and molecular-scale perspectives, we hypothesize that both kinds of mechanisms must be working in concert to drive cell rearrangement rates in confluent tissues.

While vertex models have been successful in predicting rearrangement rates in many cases, standard versions of vertex models are missing the fact that specific molecular cascades and triggers are needed to allow rearrangements. Specifically, standard vertex model formulations require that cell neighbor exchanges proceed instantaneously af- 
ter the creation of a higher-fold coordinated vertex. In systems where molecular mechanisms delay cell neighbor exchanges after the creation of higher-fold vertices, vertex models will make incorrect predictions for the global tissue response.

An extreme example is the amnioserosa tissue that is required for proper germband extension in Drosophila 24]; although the cell shapes become extremely elongated in the tissue [25], and standard vertex models would predict high numbers of cell rearrangements in response, experiments demonstrate that cells do not change their neighbor relationships at all. This is important for amniocerosa function: an elastic response generated when cells maintain neighbor relationships allows the amniocerosa to pull strongly on the germband tissue and elongate it 24, 26, 27.

While experimental work is ongoing to understand the precise molecular mechanisms that prevent cell rearrangements in the amnioserosa, there have been recent attempts to augment vertex models by incorporating various molecular mechanisms that affect how cells exchange neighbors. One model already highlighted above studies how a stress or strain threshold for $\mathrm{T} 1$ transitions affects cell shapes and rates of cell rearrangement [16]. Other models incorporate strongly fluctuating line tensions [28, 29] that can trap edges so they cannot execute $\mathrm{T} 1$ transitions.

In this manuscript, we instead augment vertex models with a model parameter we term the "T1 delay time", which is intended to incorporate a broad range of molecular mechanisms that act as a brake on $\mathrm{T} 1$ transitions. For every situation where the cell-scale dynamics generate a four-fold or higher coordinated vertex, the vertex is prevented from undergoing a $\mathrm{T} 1$ transition for a duration we call the $\mathrm{T} 1$ delay time. A similar mechanism has also been studied in independent concurrent work by Das et al [30], which focuses on how controlled T1 timescales generate intermittency and streaming states in glassy isotropic tissues. Here, we study how such a mechanism alters the global response of a tissue, both in isotropic tissues and in tissues where there is a global anisotropic change to tissues shape, such as during convergent extension in development.

We find that the "molecular-scale" T1 delay timescale dominates over the "cell-scale" collective response timescale when the T1 delay timescale is the larger of the two, slowing down tissue dynamics and solidifying the tissue. In addition, we find that increasing $\mathrm{T} 1$ delay time enhances the rosette formation in anisotropic systems. This suggests that organisms might utilize specific molecular processes that act as a brake on the resolution of four-fold and higher coordinated vertices in order to control the global tissue response in processes such as wound healing and convergent extension.

\section{MODEL}

Vertex model with $\mathbf{T} 1$ rearrangement time. We introduce a new model parameter, $\mathrm{T} 1$ delay time, both in isotropic and anisotropic vertex models. A vertex model defines an epithelial tissue as confluent tiling of $N$ cells with an energy functional for the preferred geometries of the cells [17, 22, 31]

$$
E_{i}=\sum_{i}^{N} K_{A}\left(A_{i}-A_{0 i}\right)^{2}+K_{P}\left(P_{i}-P_{0 i}\right)^{2} .
$$

In this definition, both the area and perimeter of a cell act like an effective spring. Here, $A_{i}$ and $A_{0 i}$ are the actual and preferred areas of cell $i$ while $P_{i}$ and $P_{0 i}$ are the actual and preferred perimeters. Using open-source cellGPU software [32], we simulate over-damped Brownian dynamics, where the positions of the cell vertices are updated at each time step according to

$$
\Delta r_{i}^{\alpha}=\mu F_{i}^{\alpha} \Delta t+\eta_{i}^{\alpha}
$$

using a simple forward Euler method. Here $F_{i}^{\alpha}=-\nabla_{i} E$ is the force on vertex $i$ in $\alpha$ direction, $\mu$ the inverse friction, $\Delta t$ the integration time step and $\eta_{i}^{\alpha}$ is a normally distributed random force with zero mean and $\left\langle\eta_{i}^{\alpha}(t) \eta_{j}^{\beta}\left(t^{\prime}\right)\right\rangle=2 \mu T \Delta t \delta_{i j} \delta_{\alpha \beta}$. The temperature T sets a thermal noise on vertices of each cell. The integration time step is set to $\Delta t=0.01 \tau$ where $\tau$ is the natural time unit of the simulations: $\tau=1 /\left(\mu K_{A} A_{0}\right)$.

As is standard in the literature, for instantaneous cellneighbor exchanges, a $\mathrm{T} 1$ transition proceeds whenever the distance between two vertices is less than a threshold value, $l_{c}=0.04$ in natural simulation units. We have checked that our results are not sensitive to the precise value of this cutoff. We set $K_{A}=1, K_{P}=1$, $\mu=1$ and all cells to be identical so that $A_{0 i}=A_{0}$ and $P_{0 i}=P_{0}$. We nondimensionalize the length by the natural unit length of the simulations $l=\sqrt{A_{0}}$. This yields a target shape index or a preferred perimeter/area ratio $p_{0}=P_{0} / \sqrt{A_{0}}$.

The $\mathrm{T} 1$ delay time, $t_{T 1}$, is a finite $\mathrm{T} 1$ rearrangement time that acts as a brake on $\mathrm{T} 1$ transitions, which could arise from a broad range of molecular mechanisms as discussed in the introduction. While there are many ways of implementing such a feature in our model, including adding an explicit additional energy barrier to that defined in Eq11, for simplicity we add the delay directly to the system dynamics. Specifically, when the cell-scale dynamics generate a four-fold or many-fold vertex (cellular junctions that satisfy $l<l_{c}$ criteria), the vertex is prevented from undergoing a $\mathrm{T} 1$ transition for the duration of the T1 delay time, $t_{T 1}$ (Fig. $1(\mathrm{~A}, \mathrm{~B})$ ). While the edge waits for a $t_{T 1}$ time, the configuration is not on hold the system evolves according to Eq. 2 and edges can still lengthen and shorten. In particular, every edge of a cell 
is associated with two timers (one for each connected vertex) to keep track of the time delays. When the timer reaches $t_{T 1}$, if an edge $l$ is still less than a critical length $l_{c}$, the associated cell undergoes the T1 process.

Anisotropic Vertex Model. We introduce anisotropy in our model using two different sets of simulation methods, aniosotropic line tensions and shear, similar to the protocols described in 21. For the first set of simulations, we introduce an additional line tension to nearly vertically-oriented edges. This type of perturbation was first developed to model dynamic anisotropic myosin distribution due to planar cell polarity pathways in the germband extension in Drosophila [9, 33. This is implemented via a vertex model energy functional:

$E_{i}=\sum_{i}^{N} K_{A}\left(A_{i}-A_{0 i}\right)^{2}+K_{P}\left(P_{i}-P_{0 i}\right)^{2}+\sum_{<j, k>} \gamma_{<j, k>} l_{<j, k>}$.

Here, the first sum is same as Eq1 while the second sum introduces an additional anisotropic line tension, summed over all edges connecting vertices $j$ and $k . l_{<j, k>}$ is the length of the edge between vertices $j$ and $k$, and $\gamma_{<j, k>}$ is a line tension specified as

$$
\gamma_{<j, k>}=\gamma_{0} \cos \left[2\left(\theta_{<j, k>}-\phi\right)\right],
$$

where $\gamma_{0}$ is the amplitude, $\theta_{<j, k>}$ is the edge angle and $\phi$ is the angle of anisotropy. The line tension will be maximum for the edges parallel to the lines with angle $\phi$ and will be minimum for the edges that are perpendicular to $\phi$. In the following, we fix $\phi=\pi / 2$.

For the second set of anisotropic simulations, we apply an external pure shear on the simulation box which is initially a square domain with $L_{x}=L_{y}=L$. We shear the box such that $L_{x}=e^{\epsilon} L$ and $L_{y}=e^{-\epsilon} L$. Here, $\epsilon$ is the shear strain. For the initial square domain, $\epsilon=0.0$, then we increase it by $5 \times 10^{-6}$ increments at every simulation step. After each shear, we minimize the system by updating the vertex positions but keeping the box dimensions fixed.

\section{RESULTS}

Relaxation time of the tissue. To quantify the global mechanical properties of the tissue, we characterize a relaxation time $\left(\tau_{\alpha}^{S}\right)$ as a function of $\mathrm{T} 1$ delay time $t_{T 1}$ and target shape index $p_{0}$ using the decay of a selfoverlap function. The self-overlap function is a standard correlation function used to quantify glassy dynamics in molecular and colloidal materials [34]. It represents the fraction of particles (vertices) that have been displaced by more than a characteristic distance $a$ in time t,

$$
Q_{s}(t)=\frac{1}{N} \sum_{i=1}^{N} w\left(\left|\mathbf{r}_{i}(t)-\mathbf{r}_{i}(0)\right|\right)
$$

where $\mathbf{r}_{i}$ is the position of vertex $i$ and the function $w$, $w(r \leq a)=1$ and $w(r>a)=0$. The characteristic relaxation time of the system, $\tau_{\alpha}^{S}$, is the time which most of the vertices are displaced more than a characteristic distance: $Q_{s}\left(\tau_{\alpha}^{S}\right)=1 / e$.

We run simulations across a three-dimensional parameter space: T1 delay time $t_{T 1}$, target shape index parameter $p_{0}=P_{0} / \sqrt{A_{0}}$, and temperature $T$, with 100 independent simulations initialized from different configurations for each point in parameter space. All simulations are thermalized at their target temperature for $10^{4} \tau$ before recording the data. We then run simulations for additional $3 \times 10^{5} \tau$ to ensure the system reaches a steady state.

We first use the self-overlap function to compute the characteristic relaxation time of the tissue, $\tau_{\alpha 0}^{S}$, in the absence of any T1 delays. This is simply the time at which the self-overlap function decays to $1 / e$ of its original value for simulations where the $\mathrm{T} 1$ rearrangement is instantaneous, $t_{T 1}=0$. Therefore, $\tau_{\alpha 0}^{S}$ is the typical collective response timescale that depends on cell shape and alignment in vertex models. Fig. 1(C) shows this timescale as a function of the target shape parameter $p_{0}$ with $T=0.02$. As expected, $\tau_{\alpha 0}^{S}$ decreases monotonically as $p_{0}$ increases, demonstrating that lower values of $p_{0}$ are associated with glassy behavior and increasing relaxation times.

Next, we study the behavior of the self-overlap function as a function of the $\mathrm{T} 1$ delay time. Fig. 1(D) shows that the self-overlap function changes a function of the $\mathrm{T} 1$ delay time, with $t_{T 1}=$ $0,0.13,0.46,1.67,5.99,21.5,77.4,278.2$ and $1000 \tau$ (dark green to yellow) for fixed $p_{0}=3.74$ and $T=0.02$. From this data and additional simulations at other values of $p_{0}$, we extract the characteristic characteristic relaxation time in the presence of $\mathrm{T} 1$ delays, $\tau_{\alpha}^{S}$. The inset to Fig. 1(E) shows the behavior of $\tau_{\alpha}^{S}$ as a function of $t_{T 1}$ for different values of $p_{0}=3.74,3.76,3.78 \ldots 3.9$ (darker to light blue), $T=0.02$ and $N=256$.

As $\tau_{\alpha 0}^{S}$ represents the inherent relaxation timescale of the tissue controlled by $p_{0}$ in the absence of $\mathrm{T} 1$ delays, we attempt to collapse this data by rescaling both the T1 delay timescale $t_{T 1}$ and the observed relaxation timescale $\tau_{\alpha}^{S}$ by the inherent timescale for each value of $p_{0}$. The data collapses, showing that the mechanical properties of the tissue remain unchanged for any $t_{T 1}$ delay time below $\sim 10 \% \tau_{\alpha 0}^{S}$. For $t_{T 1} \gtrsim 10 \% \tau_{\alpha 0}^{S}$, the relaxation time increases significantly, with a slope approximately equal to unity, indicating $\tau_{\alpha}^{S} \approx t_{T 1}$ when $t_{T 1}>10 \% \tau_{\alpha 0}^{S}$. The best linear fit (Fig. S1) to this region has a coefficient $m=1.13 \sim 1$, indicating that the relaxation time is just the $\mathrm{T} 1$ delay time: $\tau_{\alpha}=t_{T 1}$. This suggests that when the "molecular-scale" T1 delay timescale is larger than the cell-scale collective timescale $\tau_{\alpha 0}^{S}$, it dominates the response and solidifies the tissue. Moreover, the data collapse suggests that the cellular rearrangement timescale 
bioRxiv preprint doi: https://doi.org/10.1101/2021.02.13.431087; this version posted April 17, 2021. The copyright holder for this preprint (which was not certified by peer review) is the author/funder, who has granted bioRxiv a license to display the preprint in perpetuity. It is made available under aCC-BY-NC-ND 4.0 International license.

$\tau_{\alpha 0}^{S}$ is a good proxy for the mechanical response of the tissue over a wide range of model parameters. We note

that these results are not dependent on the system size, as shown in Fig. S2,
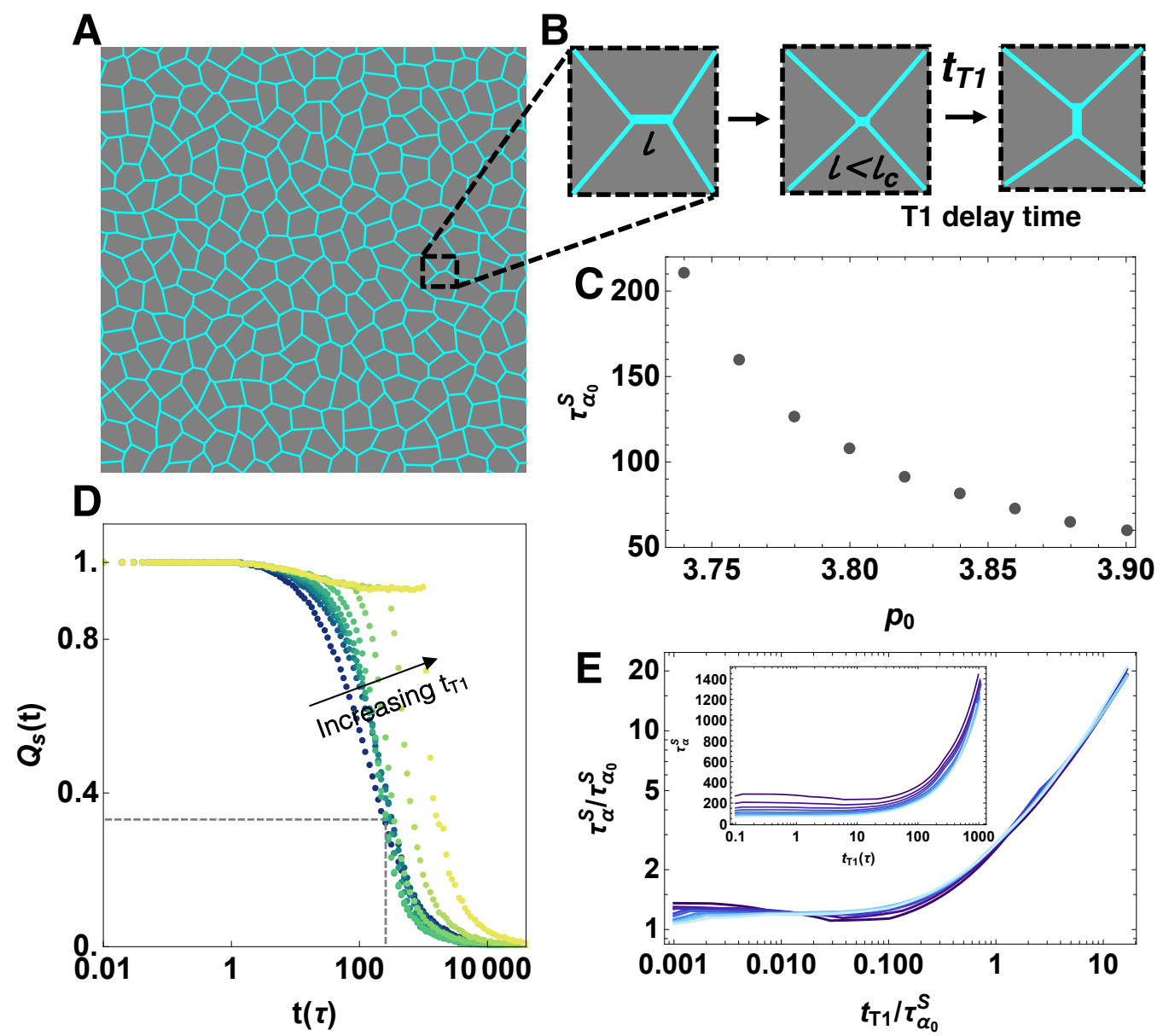

FIG. 1. Mechanical response of the isotropic vertex model at finite temperature as a function of T1 delay time A) Example cell configuration in an isotropic vertex model at a finite temperature $T=0.02$ and fixed system size $N=256$. B) Schematic of a cellular rearrangement. An edge length of $l$ shrinks to a length less than a critical length $l_{c}$, forming a four-fold vertex. The edge is prevented from undergoing the T1 transition for a $t_{T 1}$ delay time as described in the main text. C) The characteristic relaxation time in the absence of T1 delays, defined by the self-overlap function, for various $p_{0}$ values. The tissue becomes more viscous as $p_{0}$ decreases at fixed temperature. D) Self-overlap function for T1 rearrangement delay time of $t_{T 1}=0,0.13,0.46,1.67,5.99,21.5,77.4,278.2$ and $1000 \tau$ (darker green to yellow) for $p_{0}=3.74$. The dotted lines indicate where $Q_{s}\left(\tau_{\alpha 0}^{S}\right)=1 / e$ in the absence of a T1 delay. E) Log-log plot showing collapse of the characteristic relaxation time $\tau_{\alpha}^{S}$ as a function of T1 delay time normalized by the collective response timescale $\tau_{\alpha 0}^{S}$ without a T1 delay. Colors correspond to different values of $p_{0}=3.74,3.76,3.78 \ldots 3.9$ (darker to light blue), for fixed $T=0.02$, and $N=256$. The inset shows the characteristic relaxation time $\tau_{\alpha}^{S}$ as a function of $\mathrm{T} 1$ delay time without any normalization, for the same values of $p_{0}$.

Convergent extension rate is disrupted by $\mathrm{T} 1$ rearrangement time. As many biological processes require large-scale, anisotropic changes in tissue shape, we next focus on the role of $\mathrm{T} 1$ delays in models that are anisotropic.

We first study the dynamics of a vertex model with anisotropic line tensions, described by Eq. 3 and 4 . where we initialize the simulations on a square domain. Fig. 2(A) shows snapshots of the evolution of an anisotropic tissue in our simulations.
For the anisotropic tissue, we first note that the standard overlap function $Q_{s}$ defined in Eq. 5 is not a good metric for the rheology of a tissue with global shape changes or tissue flow. This is because cells may stop overlapping their initial positions due to the macroscopic flow instead of due to local neighbor exchanges that are important for rheology. Therefore, we use a different neighbors-overlap function $Q_{n}$ [35. to capture the rheology, which represents the fraction of cells that have lost two or more neighbors in time $t$ (see Supporting Information (SI) Section 1 for further details). In isotropic systems, $Q_{n}$ and $Q_{s}$ are very similar, but $Q_{n}$ is much 
bioRxiv preprint doi: https://doi.org/10.1101/2021.02.13.431087; this version posted April 17, 2021. The copyright holder for this preprint (which was not certified by peer review) is the author/funder, who has granted bioRxiv a license to display the preprint in perpetuity. It is made available under aCC-BY-NC-ND 4.0 International license.

better at identifying rheological changes in anisotropic systems. Formally, it is defined as

$$
Q_{n}(t)=\frac{1}{N} \sum_{i=1}^{N} w
$$

where $w=0$ if a cell has lost two or more neighbors and $w=1$ otherwise. Then the characteristic relaxation time of the system measured by the nearest neighbors overlap function, $\tau_{\alpha}^{N}$, is the time when $Q_{n}\left(\tau_{\alpha}^{N}\right)=1 / e$.

As in the isotropic case, we run simulations across a range of $t_{T 1}, p_{0}$, and temperature $T$, with 100 independent simulations at each point in parameter space, and where all simulations are thermalized at their target temperature for $10^{4} \tau$. We then run simulations for additional simulation time until the box reaches to a height of about four cells to avoid numerical instabilities due to periodic boundary conditions.
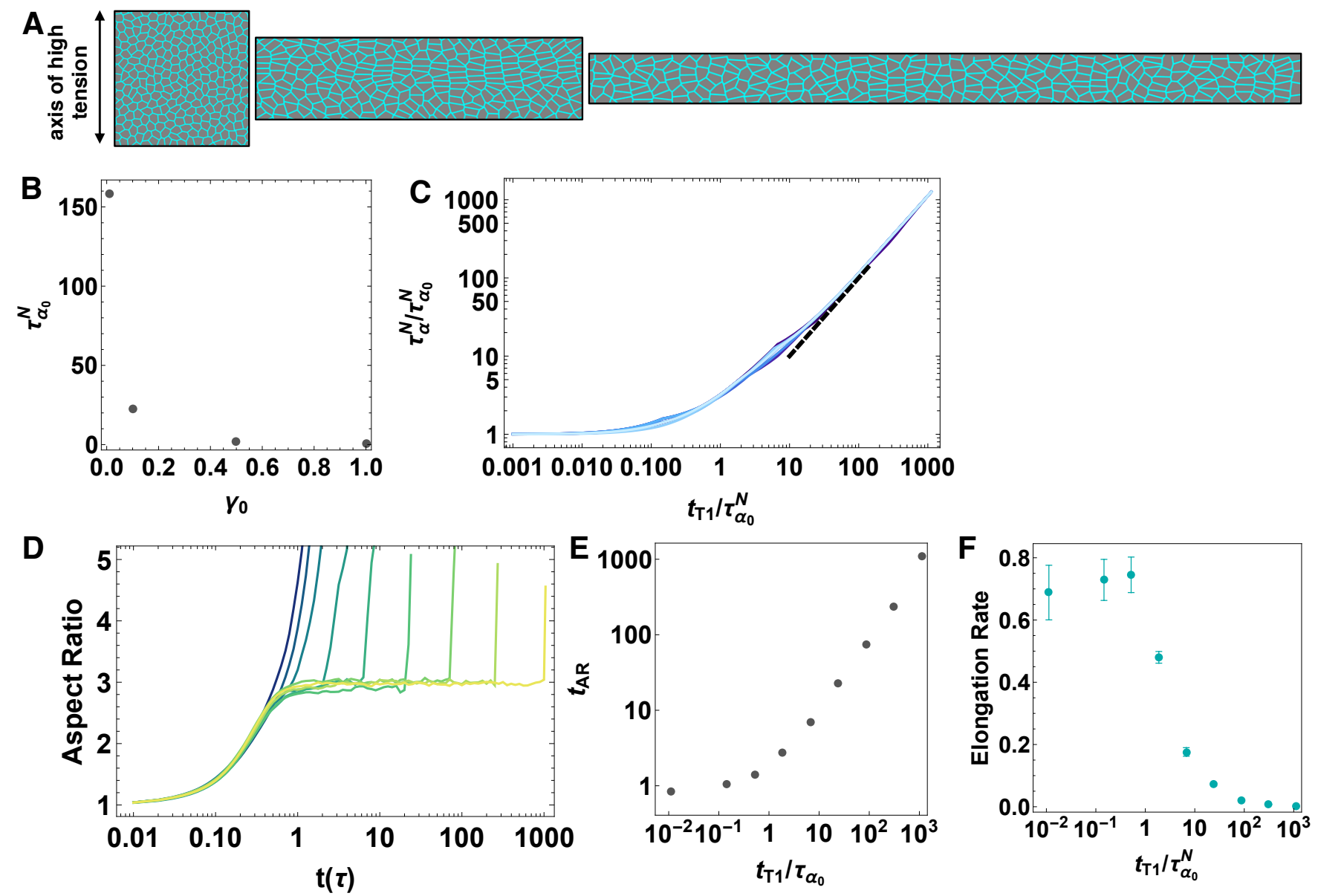

FIG. 2. Anisotropic vertex model with T1 delay time A) Simulations of an anisotropic tissue. An anisotropic line tension on vertical edges is introduced to obtain global anisotropic changes to tissue shape. B) The collective response time scale for various $\gamma_{0}$ values, the anisotropic line tension amplitude. C) Data collapse for $p_{0}=3.74 .376,3.78 \ldots 3.9$ (darker to lighter blue), $T=0.02, N=256$ and $\gamma_{0}=1.0$. The characteristic relaxation time, $\tau_{\alpha}^{N}$ as a function of T1 rearrangement delay time normalized by the collective response timescale $\tau_{\alpha 0}^{N}\left(t_{T 1}=0\right)$. The dotted line is a slope of 1 . D) The aspect ratio of the simulation box over time for T1 delay time of $t_{T 1}=0,0.13,0.46,1.67,5.99,21.5,77.4,278.2$ and $1000 \tau$ (dark green to yellow), $p_{0}=3.74, T=0.02, N=256$ and $\gamma_{0}=1.0$. E) The time $\left(t_{A R}\right)$ at which the system first goes above the plateau value as a function of $t_{T 1}$ for each aspect ratio curve in (D). F) The rate of elongation obtained from the aspect ratio curves in (D) as a function of $t_{T 1}$ delay time. (D), (E) and (F) are from 10 independent simulation runs and the rate values are average \pm one standard error.

First, we find that the characteristic relaxation $\tau_{\alpha 0}^{N}$ is controlled not only by $p_{0}$ but also by the magnitude of the applied anisotropic line tension in Eq. 4. $\gamma_{0}$. This data is shown for fixed $p_{0}=3.74$ in Fig. 2(B).

Figure 2(C) illustrates the characteristic relaxation time, $\tau_{\alpha}^{N}$, for the same values of $p_{0}$ and $T$ as shown in Fig. 1(E), but with a fixed anisotropic line tension amplitude of $\gamma_{0}=1.0$. Both axes are normalized by the collective response timescale $\tau_{\alpha 0}^{N}$ which corresponds to the case where the $\mathrm{T} 1$ rearrangement is instantaneous, 
$t_{T 1}=0$. The relation between the molecular-scale $\mathrm{T} 1$ delay timescale and collective response timescale is similar to that of the isotropic tissue, where the molecularscale T1 delay timescale dominates over the cell based collective response timescale when the $t_{T 1}$ delay time is larger than the collective response timescale. Supporting Fig. S3 shows that this result is independent of the magnitude of the line tension in the anisotropic model. We also note that the characteristic relaxation time behavior is the same at zero temperature (Fig. S4).

Next we analyze the rate of convergent extension for a fixed $p_{0}=3.74$ and $\gamma_{0}=1.0$ value, in order to study the role of T1 delays on tissue-scale deformations. Fig. 2(D) is a plot of the aspect ratio of the simulation box over time for different values of $t_{T 1}$. We see that for $t_{T 1} \lesssim \tau_{\alpha 0}^{N}$, there is a smooth elongation process until the simulation ends, consistent with a fluid-like response (or like the behavior of a yield-stress solid above the yield stress.) In this regime, increasing $t_{T 1}$ increases the rate of elongation slightly. In contrast, for $t_{T 1} \gtrsim \tau_{\alpha 0}^{N}$, the system first plateaus at a specific aspect ratio (which is about three for the parameter values shown here), and only begins to elongate beyond that value for timescales greater than $t_{T 1}$. The plateau value occurs in the absence of any rearrangements, so it is entirely due to changes in individual cell aspect ratios. It is therefore governed by a balance between $\gamma_{0}$ and $k_{P} P_{0}$, and can be predicted analytically, as described in the SI Section 3. These features are not strongly dependent on system size, as shown in (Fig. S5(D)).

The change in behavior at $t_{T 1} \sim \tau_{\alpha 0}^{N}$ is highlighted in the inset of Fig. 2(E), where we plot the time $\left(t_{A R}\right)$ at which the system first goes above the plateau value as a function of $t_{T 1}$. Similar features can be seen in a plot of the elongation rate as a function of $\mathrm{T} 1$ delay time, shown in Fig. 2(F). We calculated the rate of elongation (Fig. 2(F)) as the growth constant of an exponential fit to the aspect ratio over time (Fig. 2(D)) for each T1 delay time value.

To see if these observations are specific to systems where the anisotropy is generated by internal line tensions, or instead a generic feature of anisotropic systems, we study a vertex model in the presence of an externally applied pure shear strain (Fig. 3(A)). Fig. 3 shows the collective response of the tissue to pure shear. Fig. 3(B) illustrates the inherent relaxation timescale extracted from $Q_{n}$ as a function of $p_{0}$, and the fact that is very similar to that in Fig. 1.(C) suggests both that $Q_{n}$ and $Q_{s}$ are providing similar information and that the tissue rheology is robust across different perturbations (fluctuations vs. shear). Fig. 3(C) is similar to both Fig. 2(C) and Fig. 1.E), confirming that our observation that $\mathrm{T} 1$ delays do not affect the tissue mechanics until $t_{T 1} \sim 10 \% \tau_{\alpha_{0}}$, and beyond that value the tissue mechanics is dominated by that timescale, is robust across all perturbations studied.
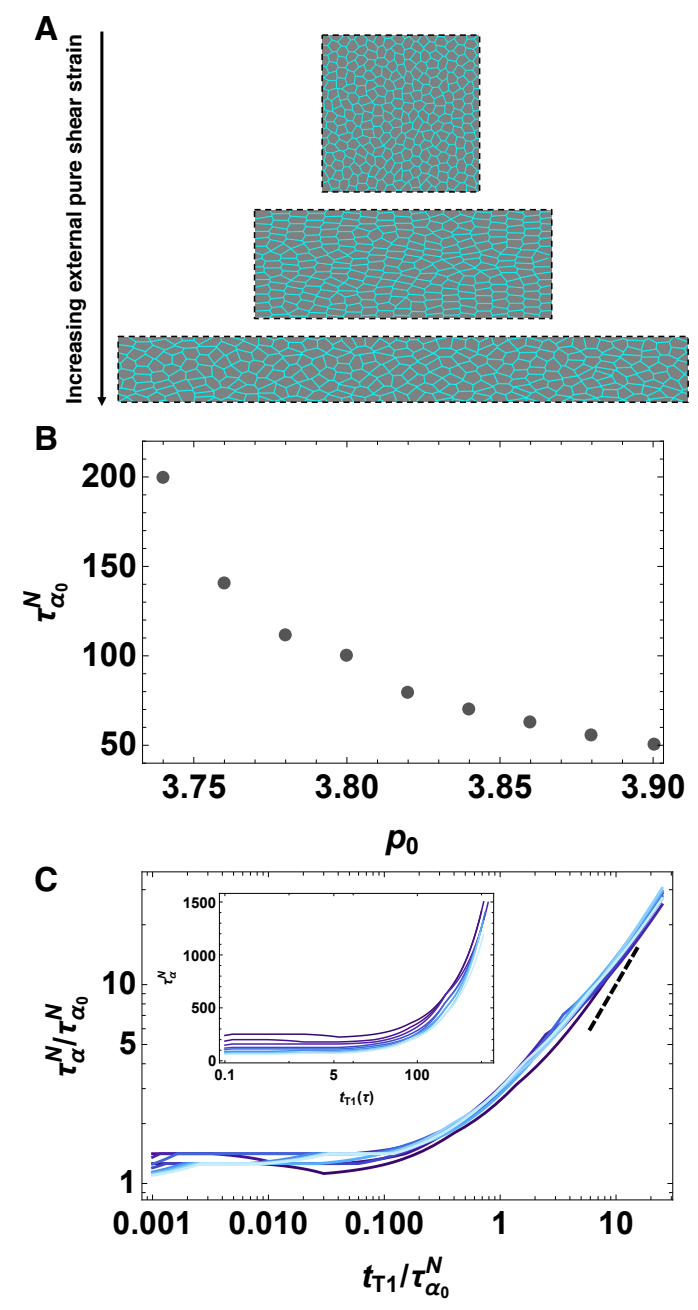

FIG. 3. Anisotropic vertex model with external pure shear and T1 delay time A) We apply an external pure shear on the simulation box which is initially a squared domain of $L_{x}=L_{y}=L$. We shear the box such that $L_{x}=e^{\epsilon} L$ and $L_{y}=e^{-\epsilon} L$. Snapshots are from simulations with $p_{0}=3.74, T=0.02, N=256$ and $t_{T 1}=1000 \tau$. B) The collective response time scale $\tau_{\alpha_{0}}^{N}\left(t_{T 1}=0\right)$ for various $p_{0}$ values. C) Data collapse for $p_{0}=3.74 .376,3.78 \ldots 3.9$ (darker to lighter blue), $T=0.02$ and $N=256$. The characteristic relaxation time, $\tau_{\alpha}^{N}$ as a function of $\mathrm{T} 1$ rearrangement delay time normalized by the collective response timescale $\tau_{\alpha 0}^{N}\left(t_{T 1}=0\right)$. The dotted line is a slope of 1 . Inset shows $\tau_{\alpha}^{N}$ the characteristic relaxation time as a function of $\mathrm{T} 1$ delay time before normalization for $\mathrm{p} 0=3.74,3.76,3.78 \ldots 3.9$ (darker to light blue).

T1 rearrangement time delays and tissue anisotropies contribute to rosette formation. While T1 transitions are the simplest type of rearrangements in epithelial monolayers, it is common for vertices that connect more than 4 cell edges to appear during developmental processes. These are termed "rosettes" and they appear often in tissue morphogenesis [4, 5] and collective cell migration [36. 
Although our model only allows three-fold coordinated vertices, previous work by some of us has shown that vertices connected by short edges can be considered as a proxy for higher-order coordinated vertices [29]. In that work, a cutoff of $0.04 \sqrt{A_{0}}$ was used to threshold very short edges as a proxy for multi-fold coordination. Something similar is also explicitly the case in experiments, where due to microscope resolution it is not possible to distinguish between very short edges and multi-fold coordinated vertices [21. In that work, a cutoff of $0.11 \sqrt{A_{0}}$ was imposed by microscope resolution, and also adopted in analysis of vertex models. Moreover, many-fold vertices are shown to be stable at heterotypic interfaces [37.

In our simulations of tissues with anisotropic line tensions, either the additional anisotropic tension on interfaces, or the edges that are prevented from undergoing $\mathrm{T} 1$ transitions, or both, could generate an increase in the number of observed rosettes. Therefore, to study the role of T1 time delays on higher-order vertex formation, we analyze the number of very short edges per cell over time in our simulations. For figures in the main text we adopt the larger cutoff of $0.11 \sqrt{A_{0}}$ used in 21, but in the supporting information (Fig. S6) we show that the results remain qualitatively similar for the smaller cutoff of $0.04 \sqrt{A_{0}}$ used in [29, although of course the overall number of very short edges is smaller with the lower threshold.
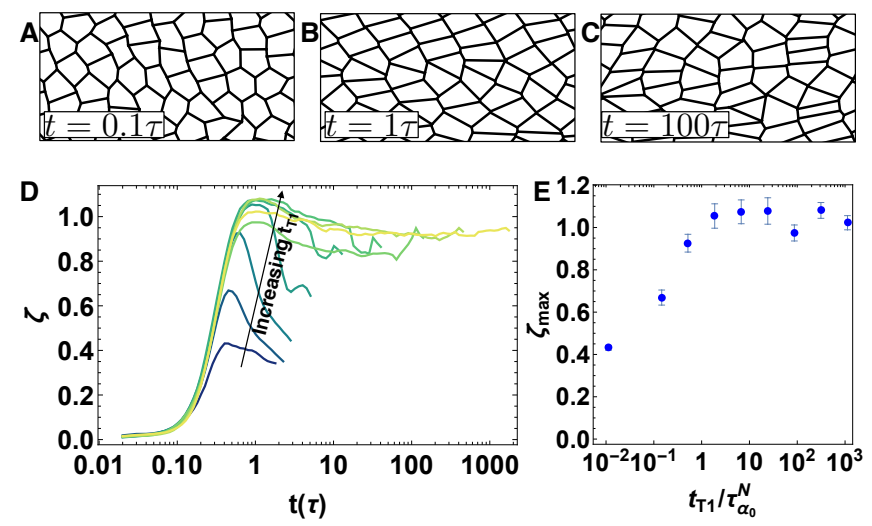

FIG. 4. Counting very short edges as a proxy for many-fold vertices Snapshots of configurations at (A) $t=$ 0.1 , (B) $t=1$, and (C) $t=100 \tau$ for $t_{T 1}=77.4 \tau$ for a tissue with $p_{0}=3.74, T=0.02, N=256$ and $\gamma_{0}=1.0$. D) Number of very short edges per cell $\xi$ for an anisotropic tissue as a function of time. Shaded lines represent different T1 delay times $t_{T 1}=0,0.13,0.46,1.67,5.99,21.5,77.4,278.2$ and $1000 \tau$ (dark green to yellow), for a tissue with $\tau_{\alpha 0}^{N}=0.89 \tau$ $-p_{0}=3.74, T=0.02, N=256$ and $\gamma_{0}=1.0$. E) Ensembleaveraged maximum value of $\xi$ over a simulation timecourse $\left(\xi_{\max }\right)$ vs. the T1 delay time $t_{T 1}$ normalized by $\tau_{\alpha 0}^{N}$. The average is taken over 10 independent simulations, and error bars correspond to one standard error.

Figure 4 panels (A-C) highlights snapshots of typical cellular structures at different timepoints in an anisotropic simulation with intermediate T1 delay time. Initially (panel A), cells are isotropic, and after about one natural time unit (panel B) vertically oriented edges under anisotropic tension have shrunk to near zero length, resulting in a significant number of 4 -fold coordinated vertices and elongated rectangular shapes with an aspect ratio set by a balance of anisotropic $\left(\gamma_{0}\right)$ and isotropic $\left(\kappa_{P} P_{0}\right)$ tensions, as discussed in SI Section 3. At timescales larger than the T1 delay time (panel C), $\mathrm{T} 1$ transitions allow some short edges to resolve and relax the structure.

Figure 4(D) shows the number of short edges (SE) per cell $\left(\xi=2 * N_{S E}^{a v g} / N_{\text {cell }}\right.$, where the factor of two reflects that edges are shared by two cells) over time for $\mathrm{T} 1$ delay of $t_{T 1}=0,0.13,0.46,1.67,5.99,21.5$, 77.4, 278.2 and $1000 \tau$ for an anisotropic tissue generated using anisotropic internal tensions. For all values of the T1 delay time, there is an initial sharp rise to a maximum value, followed by a decrease. This decrease suggests that, although there is a significant population of higher-fold vertices that remain unresolved when the T1 delay is large, some many-fold vertices resolve on longer timescales. This aligns well with experimental observations of germ-band extension in Drosophila, where cell junctions with dorsal-ventral orientation collapse to form higher order rosette structures and the rosettes are resolved by the extension of new junctions in anteriorposterior orientation 38.

In addition, Fig. $4(\mathrm{E})$ shows that as the $\mathrm{T} 1$ delay time increases, the maximum number of short edges increases. Again, we see that there is a change in behavior around $t_{T 1} \sim \tau_{\alpha 0}^{N}$. For $t_{T 1} \lesssim \tau_{\alpha 0}^{N}$, it is a monotonically increasing function, while for $t_{T 1} \gtrsim \tau_{\alpha 0}^{N}$, it plateaus at the same large value of about 1 edge per cell. This is consistent with our previous discussion of the mechanisms driving elongation, where we noted that for large $t_{T 1}$ there were no cellular rearrangements until $t=t_{T 1}$, and so prior to that timepoint the cells individually deform until they form a nearly rectangular lattice. A perfect rectangular lattice would have $\xi_{\max }=2$, so that two edges of the hexagon have shrunk to zero length, whereas in our disordered systems we find approximately one short edge per cell. Nevertheless, the maximum in the number of short edges is associated with these maximally deformed cell shapes.

We also find that in isotropic tissues, increasing the T1 delay times increases the number of many-fold vertices (Fig. S6 (B,C)), although the numbers per cell are much smaller than that in the anisotropic tissue, as expected.

All together, our results suggest that anisotropic line tension can collapse cell-cell junctions, resulting higher number of many-fold structures. The number of such structures increases as the T1 rearrangement time delay increases. Therefore, cellular rearrangement time could be a mechanism to regulate multicellular rosette structures during morphogenesis. 
Number of T1 rearrangements. To study the impact of $\mathrm{T} 1$ delays on number of $\mathrm{T} 1$ rearrangements, we calculate the number of successful $\mathrm{T} 1$ transitions per cell $\left(\eta=N_{T 1(i r r)}^{a v g} / N_{\text {cell }}\right)$ over time for a T1 delay of $t_{T 1}=$ $0,0.13,0.46,1.67,5.99,21.5,77.4,278.2$ and $1000 \tau$ (Fig. 5). In particular, in our model if an edge length $l$ is less than the critical length $l_{c}$ and the associated T1 delay timer reaches to zero, the edge orientation is flipped. However, the same edge can flip back and forth to its original orientation easily until its final steady state condition is obtained. Hence, we define successful T1 transitions as the arrangements that the cells rearrange and stay in their new configurations. In other words, the arrangements are irreversible (see SI Section 2 for details).
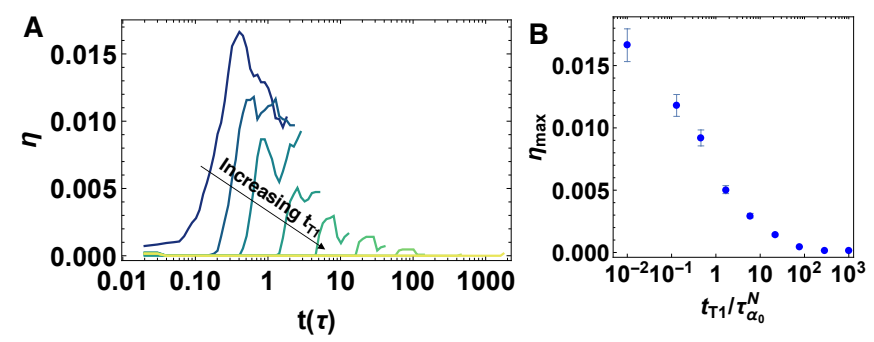

FIG. 5. Number of successful T1 transitions A) Number of successful (irreversible) $\mathrm{T} 1$ transitions per cell $\eta$ as a function of simulation time $t$ for an anisotropic tissue with $\mathrm{T} 1$ delay time of $t_{T 1}=0,0.13,0.46,1.67,5.99,21.5,77.4$, 278.2 and $1000 \tau$ (dark green to yellow), with $\tau_{\alpha 0}^{N}=0.89 \tau$ $-p_{0}=3.74, T=0.02, \gamma_{0}=1.0$ and $N=256$, averaged over 10 independent realizations. B) Number of successful (irreversible) $\mathrm{T} 1$ transitions at the maximum averaged over 10 realizations. Error bars represent one standard error.

The data for the the number of successful (irreversible) $\mathrm{T} 1$ transitions share some similarities with the analysis of short edges in 4 . Specifically Fig. 5(A) shows that the number of successful T1s grows towards a maximum and then decays, which is consistent with a picture that cells first become deformed and then execute $\mathrm{T} 1$ transitions at longer timescales to facilitate large-scale tissue deformation. Fig. 5(B) shows that this maximum decreases rapidly with increasing $\mathrm{T} 1$ delays, highlighting that the tissue response is much more elastic-like in the limit of large T1 delays. Again, we see a crossover in behavior around $t_{T 1} \sim \tau_{\alpha 0}^{N}$. Similarly, we study the effect of T1 delays on the number of $\mathrm{T} 1$ transitions for an isotropic set of simulations. The number of successful T1 transitions decreases as T1 delays increase (Fig. S7(E)), although the effect is much weaker in isotropic tissues as there are no large-scale deformations driving $\mathrm{T} 1$ transitions in that case.

Viscoelastic behavior of cellular junctions. Although the previous sections focus on global tissue response, we wanted to briefly investigate the impact of T1 delays on localized cellular junction dynamics. We apply a contractile tension on a cellular junction in a simula- tion with anisotropic internal tension. Specifically, we start from a configuration in the final steady state for a given $\gamma_{0}$ and $p_{0}$, and we choose $p_{0}=4.0$ to be in the fluid-like regime in the absence of $\mathrm{T} 1$ delays. We apply a large (Fig. 6(A)) or small (Fig. 6(B)) contractile stress on a edge, while ensuring that the stress is not large enough to generate $\mathrm{T} 1$ transitions. We then remove the stress from the edge after a fixed time period and record the global tissue response. After the stress is removed, the edge recovers a small amount but remains permanently deformed (Fig. 6(A) and (B) blue curves). We repeat the same procedure for a simulation with a large $\mathrm{T} 1$ time delay $t_{T 1}=278.2 \tau$; in this case the tissue exhibits viscoelastic features and recoils and recovers back to $\% 70-\% 90$ of its initial length in high (Fig. 6(A) orange curve) and low (Fig. 6(B) orange curve) stress cases respectively. Even though the cell shapes are similar in both cases, the local viscous response of the cellular junctions change depending on the $\mathrm{T} 1$ delays in the tissue. Again, even at this smaller scale, systems with a larger T1 delay time are more elastic while those with a smaller T1 delay are more viscous.

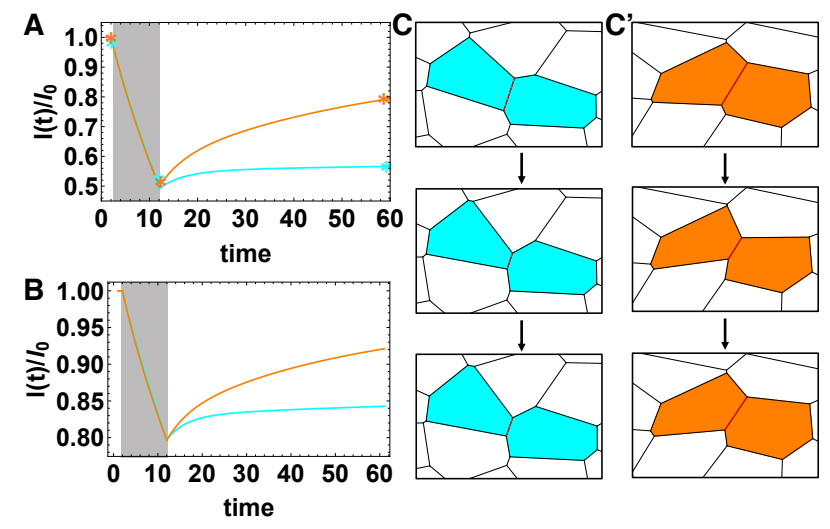

FIG. 6. Viscous response of the cellular junctions (A, B) Junction length, $l$, normalized by the initial length of the junction $l_{0}$ over time in units of simulation time steps, during and after a stress application on an edge in an anisotropic simulation. Grey regions indicate the time period of an high applied stress which shrinks the edge by \%50 (A) and a low applied stress which shrinks the edge by $\% 20$ (B). The blue curves are from the simulations without a T1 delay, $t_{T 1}=0$ and the orange curves correspond to the simulations with a T1 delay time of $t_{T 1}=278.2 \tau$. C) Snapshot from the simulations illustrating the edge dynamics before, during and after the high stress application (asterisks in (A) indicate the exact time points of the snapshots in $(\mathrm{C})$ and $\left.\left(\mathrm{C}^{\prime}\right)\right)$ with $t_{T 1}=0(\mathrm{C})$ or with $t_{T 1}=278.2 \tau\left(\mathrm{C}^{\prime}\right) \mathrm{T} 1$ delay time. Other parameters are $\gamma_{0}=1.0, p_{0}=4.0, T=0.0$ and $N=256$. 


\section{DISCUSSION AND CONCLUSIONS}

While standard vertex models for confluent tissues assume that $\mathrm{T} 1$ transitions proceed immediately after the configuration attains a multi-fold vertex, it is clear that some molecular processes may act as a brake on such transitions, generating a delay in the time required to resolve a higher-order vertex. In this work, we demonstrate that such $\mathrm{T} 1$ delays affect the tissue mechanical response in similar ways in isotropic, anisotropically sheared, and internally anisotropic tissues. Specifically, we demonstrate that the relaxation timescale associated with neighbor exchanges in the absence of $\mathrm{T} 1$ delays, $\tau_{\alpha 0}$, is an excellent metric for glassy tissue response in these disparate systems. Moreover, in systems with T1 delays, the observed relaxation timescale $\tau_{\alpha}$ is related to $\tau_{\alpha 0}$ in a remarkably simple manner. For $t_{T 1} \lesssim \tau_{\alpha 0}$, the T1 delays do not strongly affect the system and $\tau_{\alpha} \sim \tau_{\alpha 0}$, while for $t_{T 1} \gtrsim \tau_{\alpha 0}$ the $\mathrm{T} 1$ delay dominates the macroscopic dynamics and $\tau_{\alpha}=t_{T 1}$. In a related observation, we find that the number of successful T1 transitions, where cells neighbor exchange occurs and does not reverse at a later time, decreases significantly for $t_{T 1} \gtrsim \tau_{\alpha 0}$.

This suggests that in tissues where molecular mechanisms generate large $\mathrm{T} 1$ delays, the standard vertex model picture - where tissue fluidity is correlated with cell shapes, adhesion, and cortical tension - breaks down. While such molecular mechanisms are not able to speed up rearrangements, they can generically slow them down, provided that the $\mathrm{T} 1$ delays are larger that the inherent relaxation timescale of the tissue. As an example, in processes such as convergent extension of the body axis in Drosophila, the aspect ratio changes by a factor of two in about 30 minutes 8 . Given that cells in a hexagonal vertex model would normally change neighbors after a change of about two in the aspect ratio [39, 40, our results suggest that in Drosophila germband extension, molecular processes that require on the order of tens of minutes or more to complete would be effective at interfering with global extension rates.

Interestingly, the behavior of $\mathrm{T} 1$ transitions over time for large T1 delays (Fig. 5(A) exhibits similar features to those observed in the germband of Drosophila snail twist and bnt mutant embryos [21, 41. In such embryos the rearrangement rate does decrease significantly or disappear altogether, despite the fact that their cell shapes would suggest a high rearrangement rate in a standard vertex model 21. This is consistent with the hypothesis that molecular mechanisms in these mutants act as a brake on $\mathrm{T} 1$ transitions across all of developmental time.

It is interesting to speculate that even in wild type embryos, such molecular brakes could be deployed at different stages of development to "freeze in" structures sculpted previously while the tissue was a fluid-like phase. For example, after the initial rapid elongation of the body axis in fruit fly described in the previous paragraph, the cellular rearrangement rates decrease fairly precipitously about 20 minutes after the elongation process initiates, even though the cell shapes are elongated and become even more so 21.

An additional observation is that in anisotropic systems, increased T1 delay times are associated with increased persistence of higher-fold coordinated vertices, which we track by identifying very short edges in our computer model. Specifically, for $t_{T 1} \gtrsim \tau_{\alpha 0}$ we find that the number of very short edges per cell increases dramatically and remains high throughout the simulation. Again, this is consistent with the observation of a significant number of rosettes in the later stages of Drosophila body axes elongation [4].

In concurrent work, Das et al. 30 have studied a similar mechanism with an embargo on cell neighbor exchange time, for a constant target shape index parameter and in isotropic tissues. They discover interesting streaming glassy states where cells migrate in intermittent coherent streams, similar to what is seen in spheroid/ECM experiments. Our work is complementary, as we study both isotropic and anisotropic tissues over a range of shape index parameters. This allows us to emphasize the importance of the competition between the collective response timescale driven by cellscale properties and the T1 delay timescale driven by molecular scale proerties at vertices. In addition, our focus on global anisotropic changes to tissue shape allows this work to serve as a starting point for understanding how T1 delays impact developmental processes such as the convergent extension and rosette formation during body axis elongation.

Although here we use the cell neighbor exchange timescale as a read-out for tissue fluidity, an interesting avenue for future work is studying how these observations correlate with explicit mechanical measurements. As the T1 delay time we introduce via the dynamics does not alter the mechanical energy of the vertex model, infinitesimal linear response observables calculated directly from the energy, such as the bulk and shear moduli, do not include any explicit contributions from the T1 delay. As a result, the zero-temperature static shear modulus is formally zero for many of the configurations we studied, even in cases where rearrangement rates are observed to be small. A more meaningful measure would be the nonlinear and dynamic rheology of the tissue. A recent study examined the nonlinear rheology of the bare vertex model [42]; it would be interesting to extend these techniques to our system with T1 delay times. In colloids [43] it is well established that particle-motion-based measurements such as the mean squared displacement or the self-overlap function are directly related to the dynamic modulus measured in a rheometer, and it would be interesting to test this relationship in simulations and experiments on confluent tissue. 
Taken together, our work suggests that moving forward it is really important to design experiments that investigate which types of molecular processes are acting as brakes on $\mathrm{T} 1$ transitions. Obvious candidates are players in the cooperative disassembly and reassembly of complex adhesive cell-cell junctions such as adherens junctions 44] and/or desmosomes 45, or dynamics of molecules such as tricellulin that localize to three-fold coordinated vertices [12].

In recent work, Finegan et al. 14 study sdk mutants that lack the adhesion molecule Sidekick(Sdk) which localizes at tricellular vertices. They show that Drosophila sdk mutants exhibit a 1-minute delay in cell rearrangement timescales compared to wt embryos during Drosophila axis extension, accompanied by more elongated cell shapes during the extension. In addition, they develop a vertex model that explicitly allows the formation of rosettes (higher fold-coordinated vertices) and additionally specifies that rosettes take longer to resolve than simple 4 -fold coordinated vertices. The model recapitulates cell shapes and global tissue deformations seen in experiments. The spirit of the vertex model in that work is very similar to the one we report here, except that we do not require any special rules for rosettes. In our model they form naturally in systems where T1 delays occur, and they take longer to resolve simply because the individual vertices that comprise them each take longer to resolve. Therefore, it would be interesting to see if $s d k$ mutants are quantitatively consistent with the model presented here, and whether one could estimate the T1 delay timescale by fitting to the model, and then look for molecular processes at the vertices that occur on that same timescale that might be driving the delay.

In related recent work, Yu and Zallen [46] study Canoe, a different tricellular junctional protein. They find that recruitment of Canoe to tricellular junctions is correlated with myosin localization during Drosophila convergent extension, and cells are arrested at four-fold vertex configurations in embryos that express vertex-trapped Canoe. The arrested cell rearrangements are 4 min longer compared to the rearrangements in wt embryos. In combination with our work, this suggests Canoe might also regulate $\mathrm{T} 1$ delays, and that tissues with perturbed Canoe dynamics or expression might another good system for testing our predictions about the relationship between $\mathrm{T} 1$ delays and global tissue mechanical properties.

Lastly, one particularly intriguing avenue suggested by recent work [16] is whether mechano-sensitive molecules may generate a stress-dependence for the $\mathrm{T} 1$ delay timescale. In other words, our work here focuses on the effects of a fixed T1 delay timescale that is independent of any local mechanical features of the cells. However, it is possible to introduce a feedback loop where T1 delays are longer or shorter depending on the magnitude of the stresses on nearby edges, mimicking the behavior of well- known catch or slip bonds except now at a cell- or tissuescale. Such feedback loops could lead to interesting patterning and dynamical behavior.

\section{ACKNOWLEDGEMENTS}

We thank Daniel Sussman, Matthias Merkel, Peter Morse, Karen Kasza, and Xun Wang for fruitful discussions. This work was supported by NIH R01GM117598 and the Simons Foundation (grant \#446222 and \#454947 to MLM).

* gonca.erdemci@utoronto.ca

$\dagger$ mmanning@syr.edu

[1] K. E. Kasza and J. A. Zallen, Dynamics and regulation of contractile actin-myosin networks in morphogenesis, Curr. Opin. Cell Biol. 23, 30 (2011)

[2] M. Merkel, R. Etournay, M. Popovic, G. Salbreux, S. Eaton, and F. Julicher, Triangles bridge the scales: Quantifying cellular contributions to tissue deformation, Phys. Rev. E 95, 32401 (2017)

[3] D. L. Weaire and S. Hutzler, The Physics of Foams (Oxford University Press, 2000).

[4] J. T. Blankenship, S. T. Backovic, J. Sanny, O. Weitz, and J. A. Zallen, Multicellular rosette formation links planar cell polarity to tissue morphogenesis, Developmental Cell 11, 459 (2006).

[5] M. Harding, H. F. McGraw, and N. A., The roles and regulation of multicellular rosette structures during morphogenesis, Development 141, 2549 (2014).

[6] Z. J. A., Planar polarity and tissue morphogenesis, Cell 129, 1051 (2007)

[7] L. V. Goodrich and D. Strutt, Principles of planar polarity in animal development, Development 138, 1877 (2011)

[8] K. E. Kasza, D. L. Farrell, and J. A. Zallen, Spatiotemporal control of epithelial remodeling by regulated myosin phosphorylation, PNAS 111, 11732 (2014)

[9] R. J. Tetley, G. B. Blanchard, A. G. Fletcher, R. J. Adams, and B. Sanson, Unipolar distributions of junctional myosin ii identify cell stripe boundaries that drive cell intercalation throughout Drosophila axis extension, eLife 5, e12094 (2016)

[10] M. Rauzi, P. F. Lenne, and T. Lecuit, Planar polarized actomyosin contractile flows control epithelial junction remodelling, Nature 468, 1110-1114 (2010)

[11] H. Katsuno-Kambe and A. S. Yap, Endocytosis, cadherins and tissue dynamics, Traffic 21, 268 (2020).

[12] J. Ikenouchi, M. Furuse, K. Furuse, H. Sasaki, S. Tsukita, and S. Tsukita, Tricellulin constitutes a novel barrier at tricellular contacts of epithelial cells, Journal of Cell Biology 171, 939 (2005)

[13] Y. Oda, T. Otani, J. Ikenouchi, and M. Furuse, Tricellulin regulates junctional tension of epithelial cells at tricellular contacts through cdc42, Journal of Cell Science 127, 4201 (2014)

[14] T. M. Finegan, N. Hervieux, A. Nestor-Bergmann, A. G. Fletcher, G. B. Blanchard, and B. Sanson, The tricellular 
vertex-specific adhesion molecule sidekick facilitates polarised cell intercalation during drosophila axis extension, PLOS Biology 17, 1 (2019).

[15] H. Uechi and E. Kuranaga, The tricellular junction protein sidekick regulates vertex dynamics to promote bicellular junction extension, Developmental Cell 50, 327 (2019)

[16] M. F. Staddon, K. E. Cavanaugh, E. M. Munro, M. L. Gardel, and S. Banerjee, Mechanosensitive junction remodeling promotes robust epithelial morphogenesis, Biophysical Journal 117, 1739 (2019)

17] D. Bi, J. H. Lopez, J. M. Schwarz, and M. L. Manning, A density-independent glass transition in biological tissues, Nature Physics 11, 1074-1079 (2015).

[18] M. Chiang and D. Marenduzzo, Glass transitions in the cellular potts model, EPL 116, 28009 (2016)

[19] J.-A. Park, J. H. Kim, D. Bi, J. A. Mitchel, N. T. Qazvini, K. Tantisira, C. Y. Park, M. McGill, S.-H. Kim, B. Gweon, J. Notbohm, R. Steward Jr, S. Burger, S. H. Randell, A. T. Kho, D. T. Tambe, C. Hardin, S. A. Shore, E. Israel, D. A. Weitz, D. J. Tschumperlin, E. Henske, S. T. Weiss, M. L. Manning, J. P. Butler, J. M. Drazen, and J. J. Fredberg, Unjamming and cell shape in the asthmatic airway epithelium, Nature Materials 14, 1040 (2015)

[20] J. Devany, D. M. Sussman, M. L. Manning, and M. L. Gardel, Cell cycle-dependent active stress drives epithelia remodeling, bioRxiv 10.1101/804294 (2020).

[21] X. Wang, M. Merkel, L. B. Sutter, G. Erdemci-Tandogan, M. L. Manning, and K. E. Kasza, Anisotropy links cell shapes to tissue flow during convergent extension, PNAS 117, $13541(2020)$

[22] D. Bi, X. Yang, M. C. Marchetti, and M. L. Manning, Motility-Driven Glass and Jamming Transitions in Biological Tissues, Physical Review X 6, 021011 (2016).

[23] D. M. Sussman, M. Paoluzzi, M. Cristina Marchetti, and M. Lisa Manning, Anomalous glassy dynamics in simple models of dense biological tissue, EPL 121, 1 (2018)

[24] W. T. McCleery, J. Veldhuis, M. E. Bennett, H. E. Lynch, X. Ma, G. W. Brodland, and M. S. Hutson, Elongated Cells Drive Morphogenesis in a Surface-Wrapped Finite-Element Model of Germband Retraction, Biophysical Journal 117, 157 (2019)

[25] F. Schock and N. Perrimon, Cellular processes associated with germ band retraction in drosophila, Developmental Biology 248, 29 (2002)

[26] D. P. Kiehart, J. M. Crawford, A. Aristotelous, S. Venakides, and G. S. Edwards, Cell sheet morphogenesis: Dorsal closure in drosophila melanogaster as a model system, Annu. Rev. Cell Dev. Biol. 33, 169 (2017).

[27] M. E. Lacy and M. S. Hutson, Amnioserosa development and function in drosophila embryogenesis: Critical mechanical roles for an extraembryonic tissue., Developmental Dynamics 245, 558-568 (2016)

[28] M. F. Staddon, K. E. Cavanaugh, E. M. Munro, M. L. Gardel, and S. Banerjee, Solid-fluid transition and cell sorting in epithelia with junctional tension fluctuations, Soft Matter 16, 3209 (2020)
[29] T. Yamamoto, D. M. Sussman, T. Shibata, and M. L. Manning, Non-monotonic fluidization generated by fluctuating edge tensions in confluent tissues, arXiv (2020).

[30] A. Das, S. Sastry, and D. Bi, Controlled neighbor exchanges drive glassy behavior, intermittency and cell streaming in epithelial tissues, arXiv arXiv:2003.01042 (2020)

[31] R. Farhadifar, J. C. Röper, B. Aigouy, S. Eaton, and F. Jülicher, The Influence of Cell Mechanics, Cell-Cell Interactions, and Proliferation on Epithelial Packing, Current Biology 17, 2095 (2007)

[32] D. M. Sussman, cellGPU: Massively parallel simulations of dynamic vertex models, Computer Physics Communications 219, 400 (2017)

[33] M. Rauzi, P. Verant, T. Lecuit, and P. Lenne, Nature and anisotropy of cortical forces orienting drosophila tissue morphogenesis, Nature Cell Biology 10, 1401-1410 (2008)

[34] T. Castellani and A. Cavagna, Spin-glass theory for pedestrians, J. Stat. Mech. , P05012 (2005)

[35] F. Giavazzi, M. Paoluzzi, M. Macchi, D. Bi, G. Scita, M. L. Manning, R. Cerbino, and M. C. Marchetti, Flocking transitions in confluent tissues, Soft Matter 14, 3471 (2018)

[36] G. Trichas, A. M. Smith, N. White, V. Wilkins, T. Watanabe, A. Moore, B. Joyce, J. Sugnaseelan, T. A. Rodriguez, D. Kay, R. E. Baker, P. K. Maini, and S. Srinivas, Multi-cellular rosettes in the mouse visceral endoderm facilitate the ordered migration of anterior visceral endoderm cells., PLOS Biology 10, e1001256 (2012).

[37] D. M. Sussman, J. M. Schwarz, M. C. Marchetti, and M. L. Manning, Soft yet Sharp Interfaces in a Vertex Model of Confluent Tissue, Physical Review Letters 120, 1 (2018)

[38] D. Kong, F. Wolf, and J. Großhans, Forces directing germ-band extension in Drosophila embryos., Mechanisms of Development 144, 11 (2017)

[39] S. A. Khan, Foam rheology: Relation between extensional and shear deformations in high gas fraction foams, Rheologica Acta 26, 78 (1987).

[40] D. Weaire and R. Phelan, The physics of foam, Journal of Physics: Condensed Matter 8, 9519 (1996)

[41] D. L. Farrell, O. Weitz, M. O. Magnasco, and J. A. Zallen, Segga: a toolset for rapid automated analysis of epithelial cell polarity and dynamics, Development 144, 1725 (2017).

[42] C. Duclut, J. Paijmans, M. M. Inamdar, C. D. Modes, and F. Jülicher, Nonlinear rheology of cellular networks, arXiv arXiv:2103.16462 (2021).

[43] T. G. Mason and D. A. Weitz, Optical measurements of frequency-dependent linear viscoelastic moduli of complex fluids, Phys. Rev. Lett. 74, 1250 (1995).

[44] C. D'Souza-Schorey, Disassembling adherens junctions: breaking up is hard to do, Trends in Cell Biology 15, 19 (2005)

[45] Y. Kitajima, Mechanisms of desmosome assembly and disassembly, Clinical and Experimental Dermatology 27, 684 (2002).

[46] H. H. Yu and J. A. Zallen, Abl and canoe/afadin mediate mechanotransduction at tricellular junctions, Science 370, 1 (2020) 


\section{SUPPORTING INFORMATION}

\section{The neighbors-overlap function}

We define a neighbors-overlap function $Q_{n}$, as described in the text, which represents the fraction of cells that have lost two or more neighbors in time $t$. We decided upon the cutoff value of two or more neighbors based on the following observation. We compare the results obtained from the neighbors-overlap function with the results obtained from the self-overlap function. The two should give similar results for an isotropic tissue. The characteristic relaxation time obtained using the standard self-overlap function is similar to the one obtained from the neighbors-overlap function (Fig. S8 (A)). On the contrary, a definition based on a cutoff value of losing three or more neighbors show a difference between the results obtained from the self-overlap function and that of the neighbors-overlap function (Fig. S8(B)).

\section{Irreversible $\mathrm{T} 1$ transitions}

In our model, cells go through a T1 transition whenever the edge $l$ between four neighboring cells becomes less than a critical length $l_{c}$, and the T1 delay count is reached. The same group of four cells can go back and forth between their original configuration and their after $\mathrm{T} 1$ configuration until the final steady state condition is obtained (Fig. S7 (A). Hence, these flipping events can cause overcounting of the number of true T1 transitions. To avoid this, we calculate the number of successful $\mathrm{T} 1$ events where cells rearrange and stay in their new configurations. In other words, we calculate the number of irreversible T1 events.

To calculate the irreversible events $\left(N_{\text {irr }}\right)$, we first characterize the reversible events $\left(N_{\text {rev }}\right)$ in our simulations. When four cells go through a T1 transition, the two cells share an edge lose a vertex and the two cells that are not neighbor before the T1 transition gain a vertex (Fig. S7(A)). We record the cell configurations that go through the T1 transition and scan through the rest of the simulation steps to check whether the cell configurations are ever back to their "before T1" configurations in the next $\tau+t_{T 1}$ steps. Here, $\tau$ is the natural time unit of the simulations. If the configuration is repeated, we count the event as reversible, and we call the time between the two same configurations reversibility time, $t_{R}$. As we are interested in instantaneous successful T1 events over time, we search for the reversibility in short timescales, namely in $\tau$ steps. Fig. S7.B) is a histogram of reversibility time for $t_{T 1}=0$ which fits to a power law (Fig. S7(B) inset) with a long tail which indicates that most of the flipping T1 events happen in very short timescales.

We calculate the fraction of irreversible events, $f=N_{i r r} / N_{\text {total }}$. For an anisotropic tissue, $70 \%$ of the T1 events are irreversible for $t_{T 1}=0$ while almost all of the events are irreversible for $t_{T 1}>0$ (Fig. S7(C)). For an isotropic tissue, only $20 \%$ of the T1 transitions are irreversible for $t_{T 1}=0$ (Fig. S7(D)). The irreversibility increases as the $t_{T 1}$ delay time increases (Fig. S7(D)). To calculate the successful T1 events (main text Fig. 5(A) and (B)), we multiply the total number of T1 transitions by the fraction of irreversible events $f$ (Fig. S7 (C)) at each $t_{T 1}$ value. Similarly, for an isotropic tissue (Fig. S7(E)), we multiply the total number of T1 transitions by the fraction of irreversible events $f$ (Fig. S7 (D)) at each $t_{T 1}$ value.

\section{Plateau value for the tissue aspect ratios in the absence of T1 transitions}

As discussed in the main text and seen in Fig. 2(D), in the absence of T1 transitions (i.e., at simulation timepoints before the T1 delay timescale) tissues under anisotropic tension will increase their aspect ratio up to a plateau value and get stuck there. Numerical simulations confirm that this plateau value varies with both the isotropic tension $\kappa_{P} P_{0}$ in the bare vertex model and the additional anisotropic line tension $\gamma_{0}$ (Fig. S9.

To predict this plateau value analytically, we hypothesize that the plateau occurs when the shapes are at an energy minimum under the constraint there are no T1 transitions. We propose that the cells undergo a two-step process to minimize their energy, which is easiest to see starting from an ordered isotropic hexagonal packing, shown in Supporting Fig S10(A). First, the large line tension on the vertical edges will cause those to shrink to zero length, generating a diamond pattern with 4 -fold coordinated vertices, as shown in Fig S10(B). This process should be independent of $\gamma_{0}$ and $P_{0}$ for values of $\gamma_{0}$ that are sufficiently large, and one can show it results in a change of aspect ratio by a factor of 1.58: $A R_{1}=1.58$.

Next, because that state is perfectly symmetric, the system can undergo a symmetry breaking so that some edges become shorter and closer to vertically-oriented, while alternating edges become longer and more horizontally oriented. 
In an ordered systems this generates some flag-shaped parallelograms, but in a disordered system one expects shapes that are roughly rectangular with the short sides oriented along the axis of higher tension, as shown in Fig S10(C). To calculate the minimum energy state of such rectangles, we label the vertical length $l_{1}$ and horizontal length $l_{2}$, and assume the area of each cell is fixed to unity so that $l_{2}=1 / l_{1}$. Taking the derivative of the part of the vertex energy functional related to line tensions, $E \sim \gamma_{0} l_{1}+\kappa_{P}\left(P-P_{0}\right)^{2}$, and then setting the derivative with respect to $l_{1}$ equal to zero results in a 4 th order polynomial equation:

$$
l_{1}^{4}+\left(\frac{\gamma_{0}}{8 \kappa_{P}}-\frac{P_{0}}{2}\right) l_{1}^{3}+\frac{P_{0}}{2} l_{1}-1=0 .
$$

For a given values of $\kappa_{P}, P_{0}$, and $\gamma$, we can solve this equation for its positive roots and identify the energy minimizing value of $l_{1}^{\text {min }}$. The addition change to the global aspect ratio of the tissue allowed by these rectangles is simply ratio of $l_{2}=1 / l_{1}$ to $l_{1}: A R_{2}=1 /\left(l_{1}^{\text {min }}\right)^{2}$.

Finally, the total change to the aspect ratio under both processes is simply $A R_{t o t}=A R_{1} \times A R_{2}=1.58 /\left(l_{1}^{\min }\right)^{2}$. This analytic prediction is illustrated by the blue squares in Fig S10(D). The observed plateau values are given by the red circles, and they are in fairly good agreement. The analytic prediction overestimates the aspect ratio for the lowest value of $\gamma_{0}$, likely because the tension isn't sufficiently large to shrink vertical edges to zero in the first process. 


\section{Supporting figures}

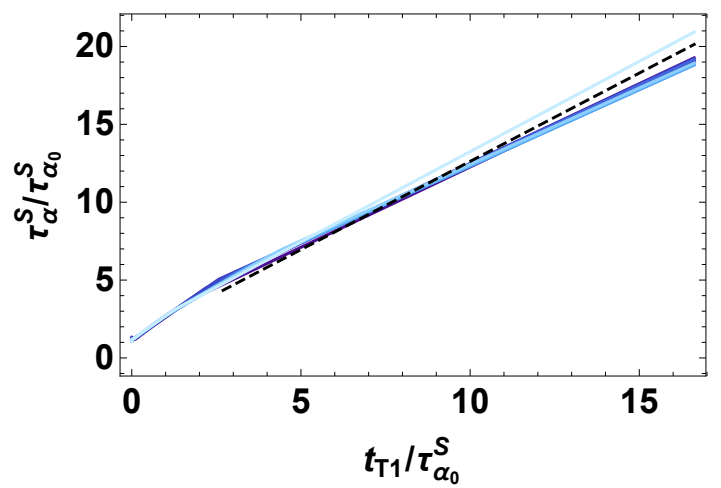

FIG. S1. The characteristic relaxation time $\tau_{\alpha}^{S}$ on linear scale (log-log scale as in Fig. 1) as a function of T1 delay time normalized by the collective response timescale $\tau_{\alpha 0}^{S}$ without $\mathrm{T} 1$ delay. The dashed line is the best linear fit to high $\mathrm{T} 1$ delay region with a slope of $m=1.13$. Colors correspond to different values of $p_{0}=3.74,3.76,3.78 \ldots 3.9$ (darker to light blue), for fixed $T=0.02$, and $N=256$.
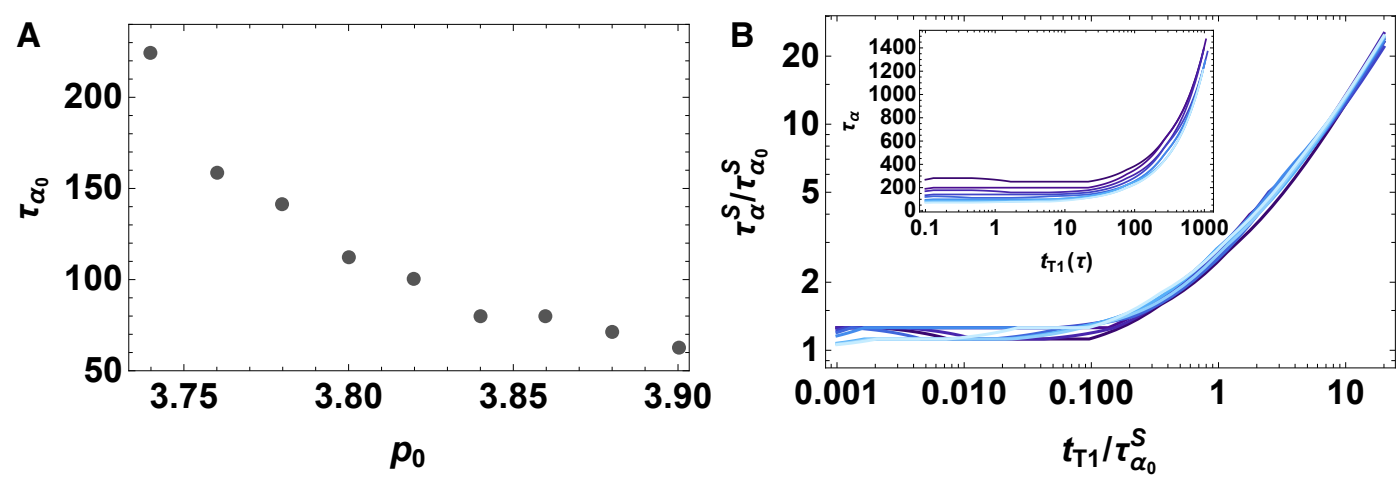

FIG. S2. A) The characteristic relaxation time in the absence of T1 delays, defined by the self-overlap function, for various $p_{0}$ values for a system size with $N=1024$. The tissue becomes more viscous as p0 decreases at fixed temperature $T=0.02$. B) Log-log plot showing collapse of the characteristic relaxation time $\tau_{\alpha}^{S}$ as a function of T1 delay time normalized by the collective response timescale $\tau_{\alpha 0}^{S}$ without a T1 delay. Colors correspond to different values of $p_{0}=3.74,3.76,3.78 \ldots 3.9$ (darker to light blue), for fixed $T=0.02$, and $N=1024$. The inset shows the characteristic relaxation time $\tau_{\alpha}^{S}$ as a function of T1 delay time without any normalization, for the same values of $p_{0}$. 
bioRxiv preprint doi: https://doi.org/10.1101/2021.02.13.431087; this version posted April 17, 2021. The copyright holder for this preprint (which was not certified by peer review) is the author/funder, who has granted bioRxiv a license to display the preprint in perpetuity. It is made available under aCC-BY-NC-ND 4.0 International license.
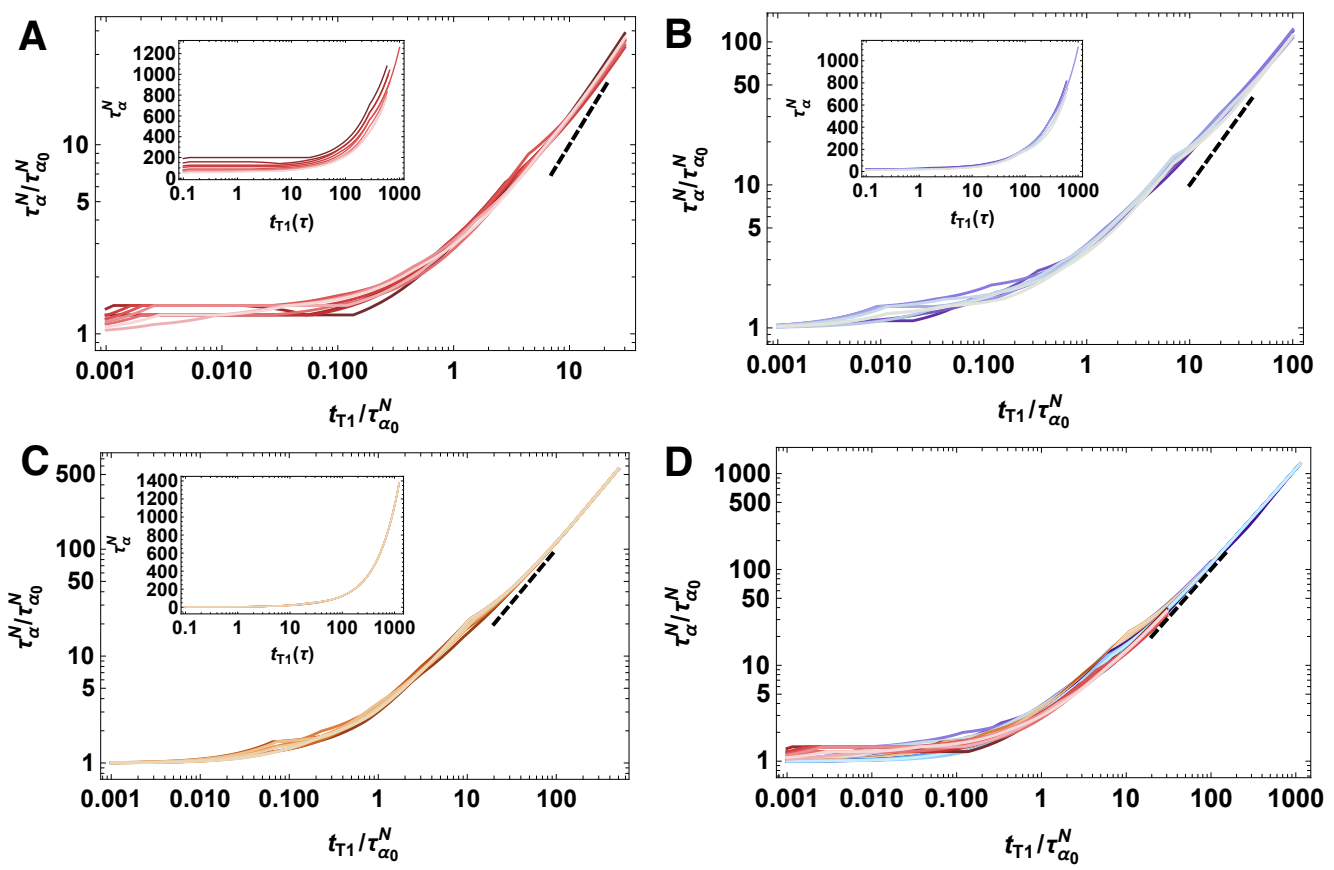

FIG. S3. A) The characteristic relaxation time $\tau_{\alpha}^{N}$ as a function of $\mathrm{T} 1$ rearrangement delay time for anisotropic tissue simulations with anisotropic line tension amplitude of $\gamma_{0}=0.01(\mathrm{~A}), \gamma_{0}=0.1(\mathrm{~B})$ and $\gamma_{0}=0.5(\mathrm{C})$. D) Overlap of the data in (A), (B), (C) and Fig 2(C). Darker to lighter tones for each color represents the data for $p_{0}=3.74,3.76,3.78 \ldots 3.9$, and other parameters are $T=0.02$, and $N=256$. Each data set are normalized by the corresponding characteristic relaxation time $\tau_{\alpha 0}^{N}$ where the $\mathrm{T} 1$ rearrangement is instantaneous, $t_{T 1}=0$.

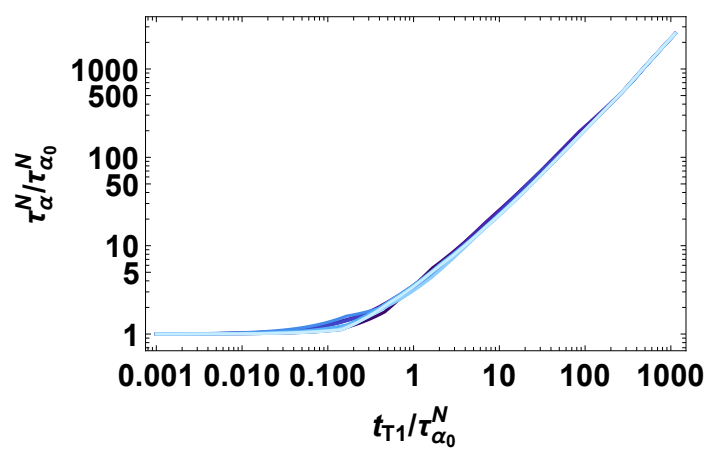

FIG. S4. Data collapse for $p_{0}=3.74 .376,3.78 \ldots 3.9$ (darker to lighter blue), $N=256$ and $\gamma_{0}=1.0$ at zero temperature. The characteristic relaxation time, $\tau_{\alpha}^{N}$ as a function of $\mathrm{T} 1$ rearrangement delay time normalized by the collective response timescale $\tau_{\alpha 0}^{N}\left(t_{T 1}=0\right)$. 
bioRxiv preprint doi: https://doi.org/10.1101/2021.02.13.431087; this version posted April 17, 2021. The copyright holder for this preprint (which was not certified by peer review) is the author/funder, who has granted bioRxiv a license to display the preprint in perpetuity. It is made available under aCC-BY-NC-ND 4.0 International license.

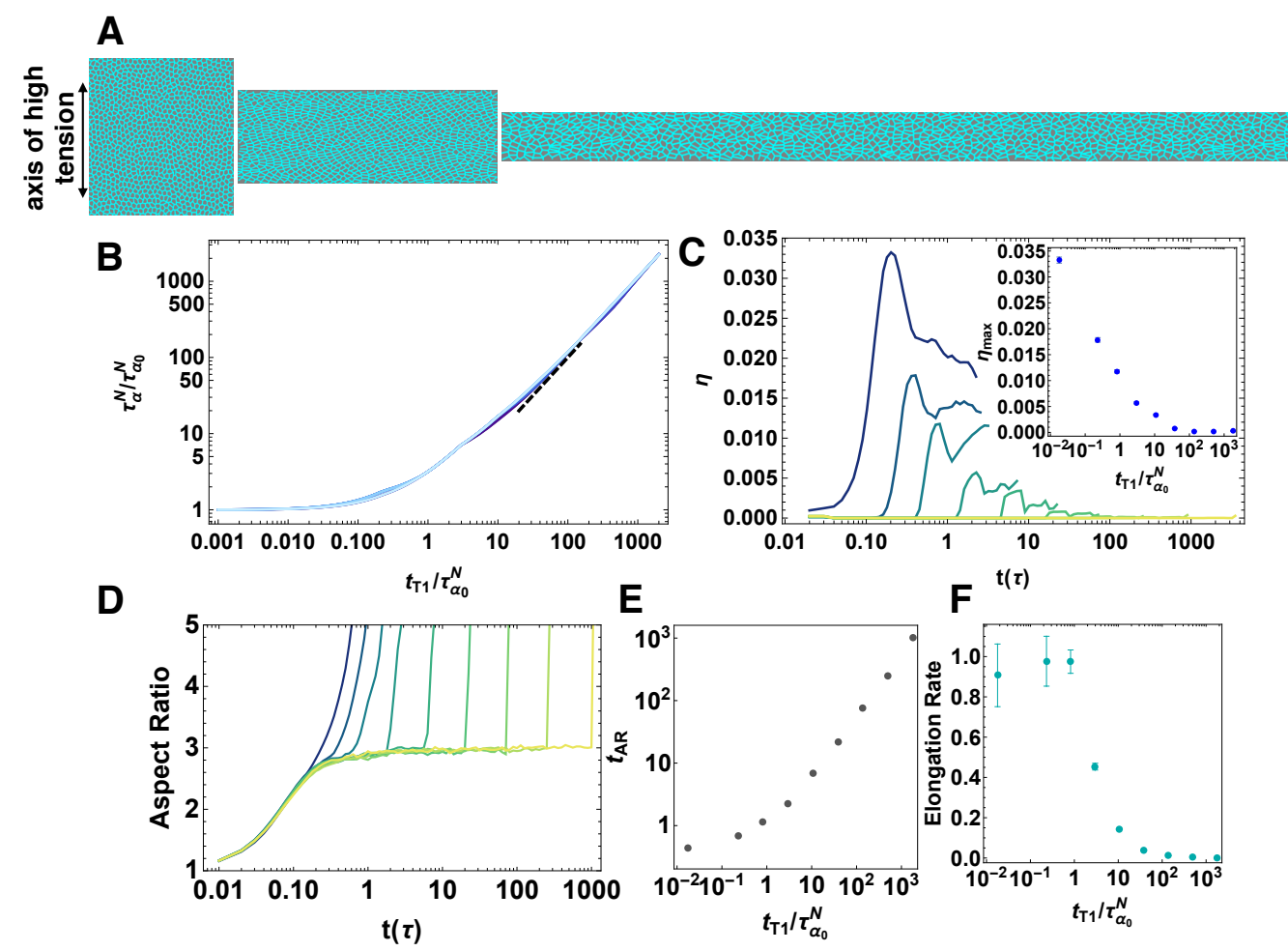

FIG. S5. A) Simulations of an anisotropic tissue with a larger size. An anisotropic line tension on vertical edges is introduced to obtain global anisotropic changes to tissue shape. B) Data collapse for $p_{0}=3.74 .376,3.78 \ldots 3.9$ (darker to light blue), $T=0.02, N=1024$ and $\gamma_{0}=1.0$. The characteristic relaxation time, $\tau_{\alpha}^{N}$ as a function of $\mathrm{T} 1$ rearrangement delay time normalized by the collective response timescale $\tau_{\alpha 0}\left(t_{T 1}=0\right)$. The dotted line is a slope of 1 . C) The number of T1 transitions per cell over time and at the maximum averaged over 10 realizations (inset) for T1 delay time of $t_{T 1}=0,0.13,0.46,1.67,5.99$, 21.5, 77.4, 278.2 and $1000 \tau$ (dark green to yellow), $p_{0}=3.74, T=0.02, N=1024$ and $\gamma_{0}=1.0$. D) The aspect ratio of the simulation box over time for $\mathrm{T} 1$ delay time of $t_{T 1}=0,0.13,0.46,1.67,5.99,21.5,77.4,278.2$ and $1000 \tau$ (dark green to yellow), $p_{0}=3.74, T=0.02, N=1024$ and $\gamma_{0}=1.0$. E) The time $\left(t_{A R}\right)$ at which the system first goes above the plateau value as a function of $t_{T 1}$ for each aspect ratio curve in (D). F) The rate of elongation obtained from the aspect ratio curves in (D) as a function of $t_{T 1}$ delay time. Both (C), (D), (E) and (F) are from 10 realizations. Error bars represent one standard error. 
bioRxiv preprint doi: https://doi.org/10.1101/2021.02.13.431087; this version posted April 17, 2021. The copyright holder for this preprint (which was not certified by peer review) is the author/funder, who has granted bioRxiv a license to display the preprint in perpetuity. It is made available under aCC-BY-NC-ND 4.0 International license.

A

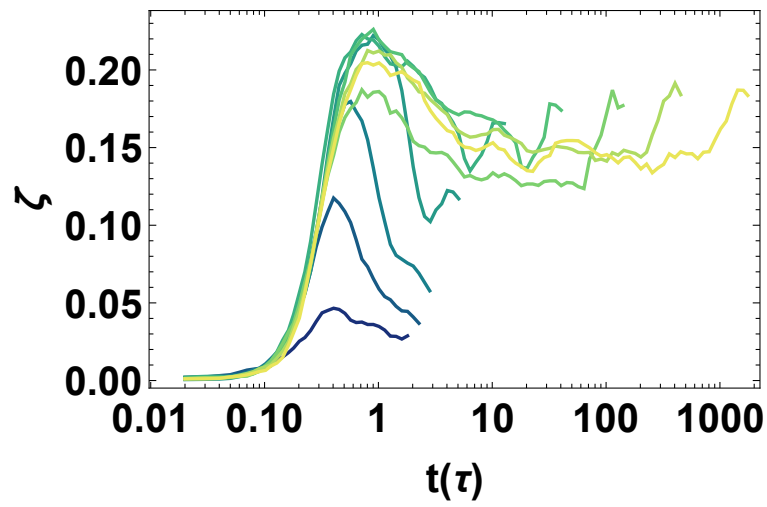

B

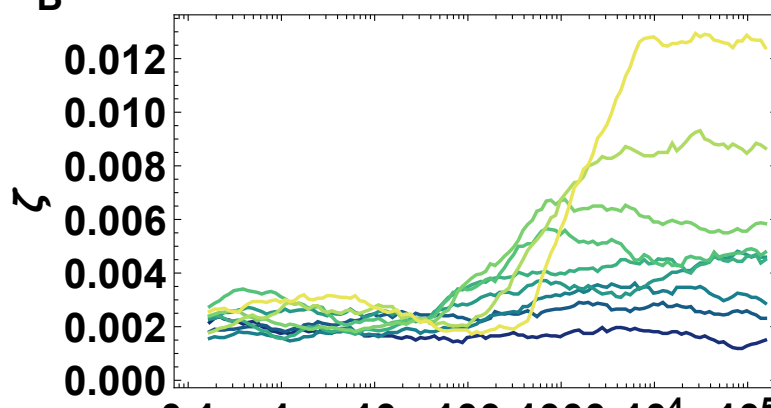

$0.1110100100010^{4} \quad 10^{5}$

C

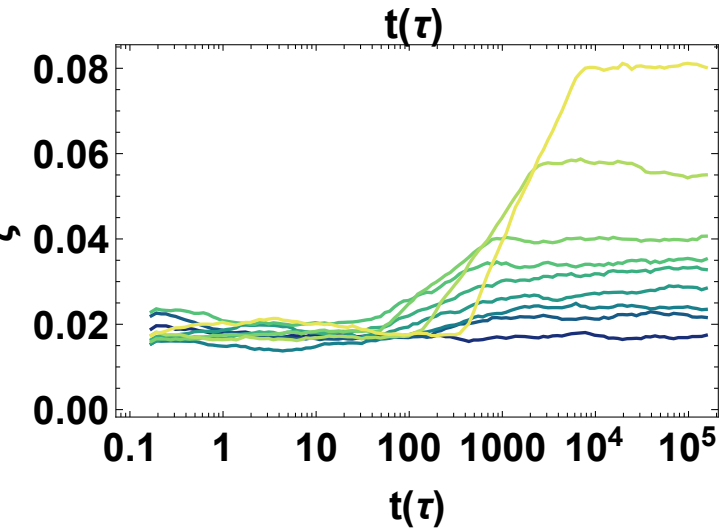

A'

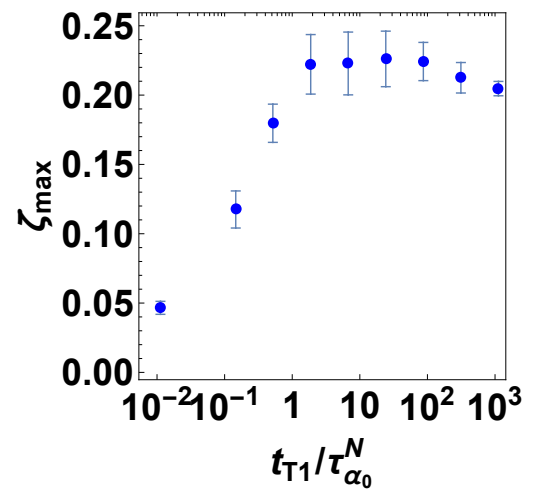

B'

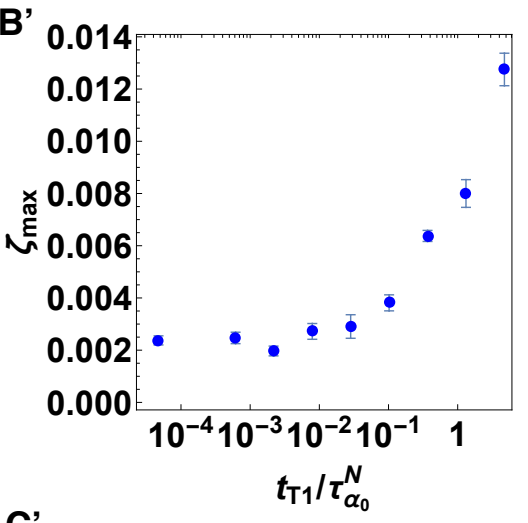

C'

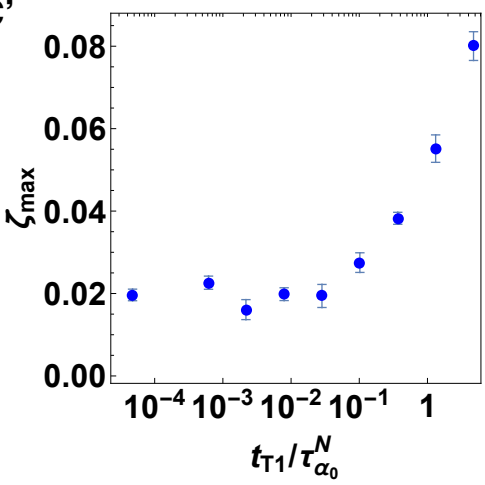

FIG. S6. Number of very short edges per cell $\xi$ for an anisotropic tissue as a function of time (A). Shaded lines represent different $\mathrm{T} 1$ delay times $t_{T 1}=0,0.13,0.46,1.67,5.99,21.5,77.4,278.2$ and $1000 \tau$ (dark green to yellow), for a tissue with $\tau_{\alpha 0}^{N}=0.89 \tau-p_{0}=3.74, T=0.02, N=256$ and $\gamma_{0}=1.0$. The cutoff value to threshold very short edges as a proxy for multi-fold coordination is $0.04 \sqrt{A_{0}}$. (A') Ensemble-averaged maximum value of $\xi$ over a simulation timecourse vs. the T1 delay time $t_{T 1}$ normalized by $\tau_{\alpha 0}^{N}$. The average is taken over 10 independent simulations, and error bars correspond to one standard error. Number of very short edges per cell $\xi$ over time (B, C) and the ensemble-averaged maximum value of $\xi$ over a simulation timecourse (B', C') for an isotropic tissue simulations of T1 delay time of $t_{T 1}=0,0.13,0.46,1.67,5.99,21.5,77.4,278.2$ and $1000 \tau$ (dark green to yellow), $p_{0}=3.74, T=0.02$ and $N=256$. The cutoff value to threshold very short edges as a proxy for multi-fold coordination is $0.04 \sqrt{A_{0}}$ in $\left(\mathrm{B}, \mathrm{B}^{\prime}\right)$ and $0.11 \sqrt{A_{0}}$ in $\left(\mathrm{C}, \mathrm{C}^{\prime}\right)$. The average is taken over 10 independent simulations, and error bars correspond to one standard error. 
bioRxiv preprint doi: https://doi.org/10.1101/2021.02.13.431087; this version posted April 17, 2021. The copyright holder for this preprint (which was not certified by peer review) is the author/funder, who has granted bioRxiv a license to display the preprint in perpetuity. It is made

A
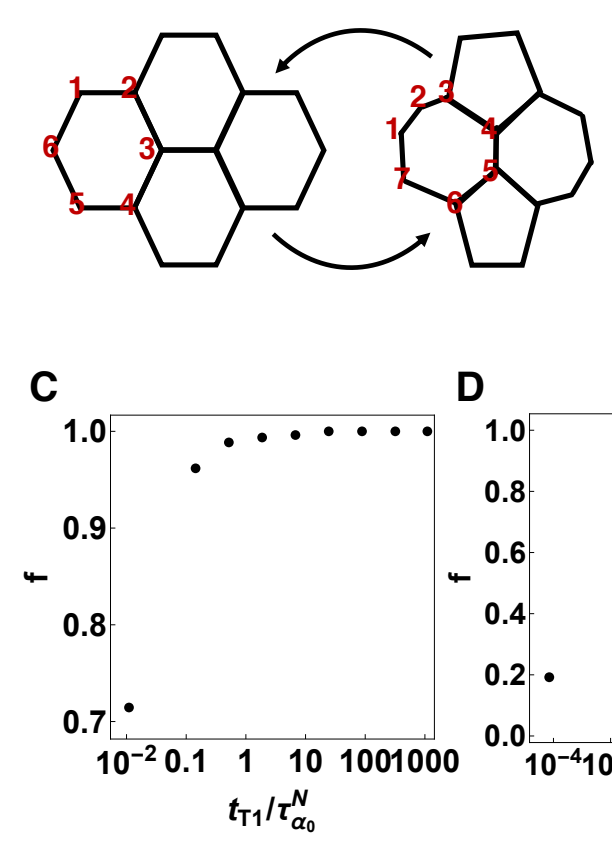

D
B

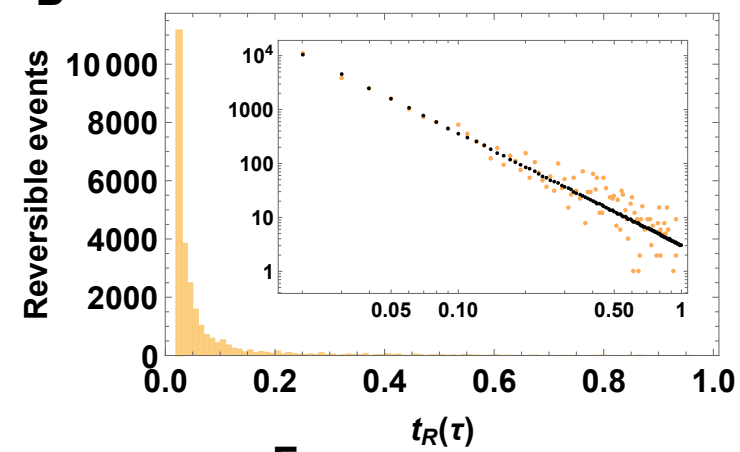

E
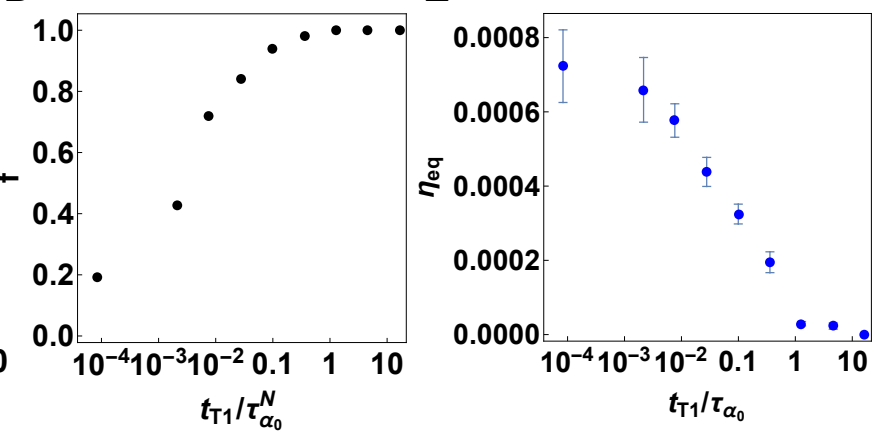

FIG. S7. A) Schematic of a reversible T1 transition. The two cells that are neighbor before the T1 transition lose a vertex (top and bottom cells) and the cells that are not neighbors before the T1 transition gain a vertex and become neighbors (right and left cells). Vertices of the cell on the left are labeled as $1,2, \ldots 6$. The same cell has a configuration of vertices as $1,2, \ldots 7$ after the $\mathrm{T} 1$ transition. If the $\mathrm{T} 1$ transition is reversible, then the cell goes back to its original configuration of vertices $1,2, \ldots 6$. We track such cell configuration changes in our simulations to determine if a T1 event is reversed. B) Histogram of reversibility time $t_{R}$ which fits to a power law (inset black points) for an anisotropic tissue of $p_{0}=3.74, T=0.02, N=256, t_{T 1}=0$ and $\gamma_{0}=1.0$. C) Fraction of irreversible events as a function of $\mathrm{T} 1$ delay time for an anisotropic tissue of $p_{0}=3.74, T=0.02$, $N=256$ and $\gamma_{0}=1.0$. D) Fraction of irreversible events as a function of T1 delay time for an isotropic tissue of $p_{0}=3.9$, $T=0.02$ and $N=256$. E) Number of successful (irreversible) T1 transitions at the equilibrium averaged over 10 realizations for an isotropic tissue of $p_{0}=3.9, T=0.02$ and $N=256$. Error bars represent one standard error.
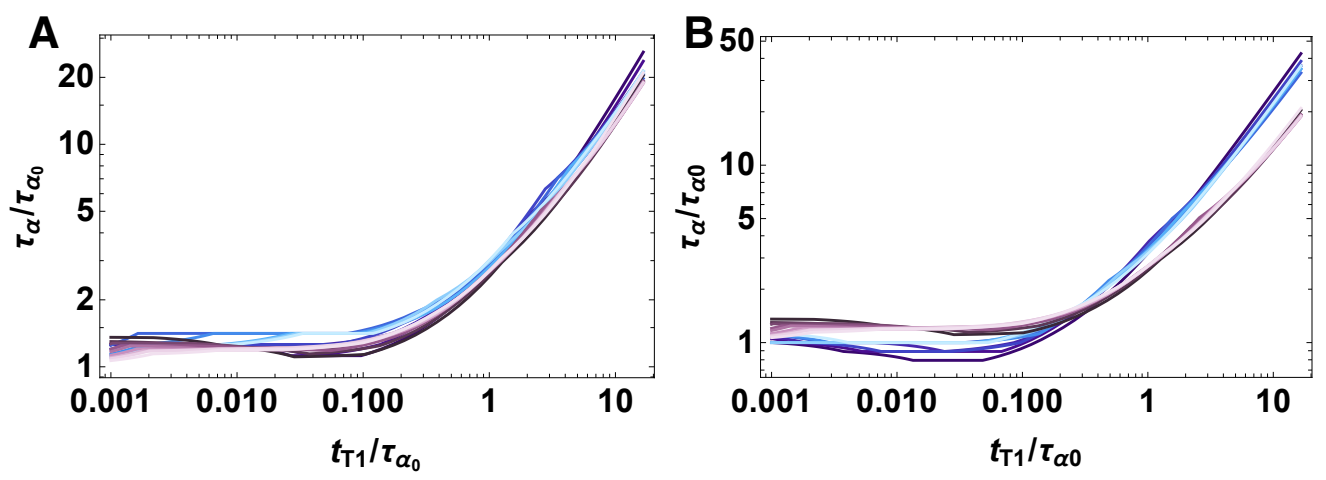

FIG. S8. The characteristic relaxation time, $\tau_{\alpha}$, obtained using neighbors-overlap function (based on losing 2 or more neighbors (A) and losing 3 or more neighbors (B)), as a function of T1 rearrangement time for $p_{0}=3.74,3.76,3.78 \ldots 3.9$ (darker to light blue) and $T=0.02$ for an isotropic tissue. Both (A) and (B) are plotted together with the characteristic relaxation time obtained using the self-overlap function for comparison for $p_{0}=3.74,3.76,3.78 \ldots 3.9$ (dark purple to light pink). Each data set are normalized by the corresponding characteristic relaxation time $\tau_{\alpha 0}$ where the $\mathrm{T} 1$ rearrangement is instantaneous, $t_{T 1}=0$. 
bioRxiv preprint doi: https://doi.org/10.1101/2021.02.13.431087; this version posted April 17, 2021. The copyright holder for this preprint (which was not certified by peer review) is the author/funder, who has granted bioRxiv a license to display the preprint in perpetuity. It is made
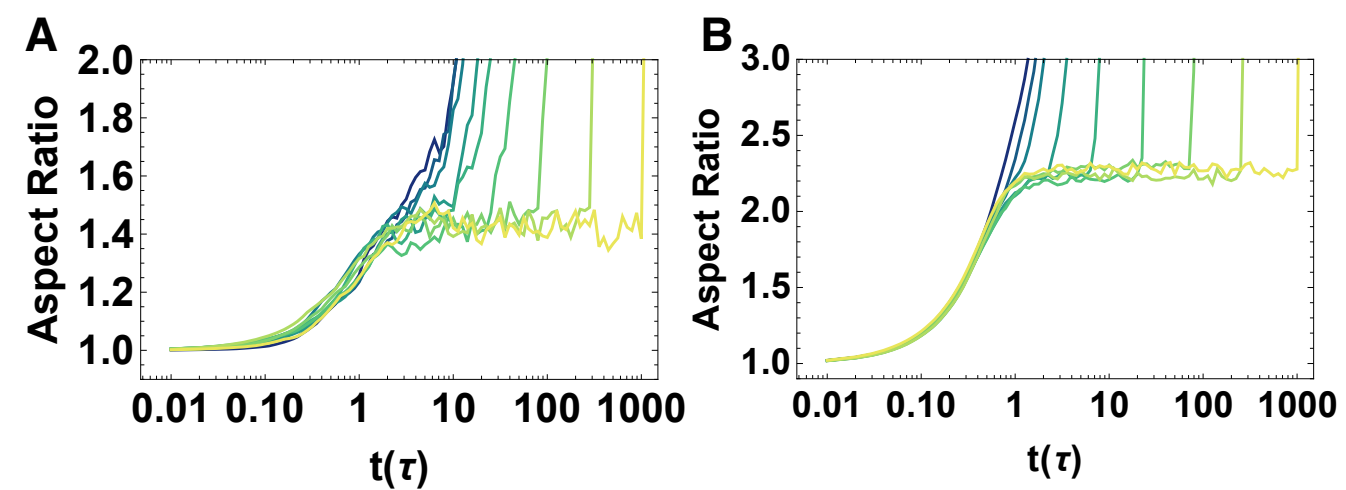

FIG. S9. The aspect ratio of the simulation box over time for $\mathrm{T} 1$ delay time of $t_{T 1}=0,0.13,0.46,1.67,5.99,21.5,77.4$, 278.2 and $1000 \tau$ (dark green to yellow), $p_{0}=3.74, T=0.02$ and $N=256$. The anisotropic line tension amplitude $\gamma_{0}=0.1$ (A) and $\gamma_{0}=0.5(\mathrm{~B})$. The data in $(\mathrm{A})$ plateau at aspect ratio of $\sim 1.4$ and the data in $(\mathrm{B})$ plateau at aspect ratio of $\sim 2.2$.
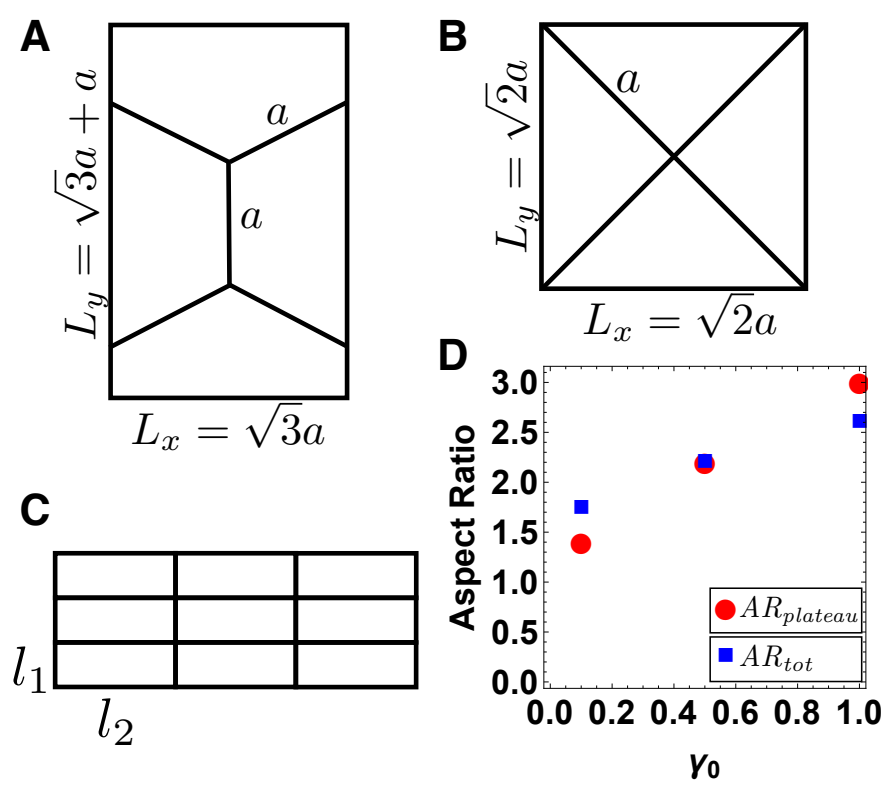

FIG. S10. A) A hexagonal unit box with a hexagon side of $a$. B) The cells meet at a 4-fold coordinated vertex after a high tension is applied on the vertical edges of the hexagons in (A). Cells form a diamond pattern with a side length of $a$. Both in (A) and (B), $L_{x}$ and $L_{y}$ are the sides of the box along the horizontal and vertical direction. C) A rectangular grid of cells with sides $l_{1}$ along the vertical and $l_{2}$ along the horizontal direction. D) Analytical prediction of the plateau values observed in simulations with various $\gamma_{0}$ values (Fig. 2D, Fig. S9A, Fig. S9B). Red circles correspond to the plateau values in the simulations. Blue squares correspond to the analytical prediction for the total change to the aspect ratio as described in SI Section 3. 